\title{
¿Qué es una Buena Elección?: El Uso de los Informes de las Misiones de Observación Electoral para Evaluar los Procesos Electorales Latinoamericanos (2013-2016)*
}

\section{Flavia Freidenberg ${ }^{1}$}

${ }^{1}$ Universidad Nacional Autónoma de México (UNAM), Cidade do México, México. E-mail: flavia@unam.mx

\section{INTRODUCCIÓN}

$\mathrm{U}$ no de los consensos de la política comparada se centra en la idea de que las elecciones son el corazón de la democracia representativa (Schattschneider, 1964; Dahl, 1971). Se entiende por elecciones democráticas a los "mecanismos de elección social [que se ejercen] en condiciones de libertad e igualdad" (Schedler, 2002). Este ha sido el paradigma dominante desde la perspectiva de la democracia elitista o pluralista (Dahl, 1971) y tuvo gran relevancia en el modo en que las élites y la ciudadanía entendieron la democracia y lucharon por la instauración de las poliarquías en América Latina, tras los procesos de transición de la tercera ola de finales de la década de 1970 (Zovatto, 2006) ${ }^{1}$.

La celebración ininterrumpida de elecciones durante los últimos cuarenta años en América Latina las convierte en "eventos rutinarios" (Mozzafar y Schedler, 2002), en "el único juego posible en la ciudad" (Linz, 1987), adquiriendo "vida propia" (Schedler, 2002). Esto ha llevado a creer que cuanto más rutinarias sean las elecciones, más institucionalizada se encuentra la democracia (Hartlyn y McCoy, 2006), en particular, cuando goza de altos niveles de "gobernanza electoral" (Lehoucq, 2002; Hartlyn et al. 2008), es decir, cuando se da una adecua-

\footnotetext{
* Agradezco los comentarios y sugerencias que Yuri Gabriel Beltrán Miranda, Gerardo de Icaza y Alberto Guevara Castro realizaron a diversas versiones de este texto así como también dos revisores anónimos de la revista. Todos sus comentarios han contribuido a mejorar los argumentos y el desarrollo de este artículo.
}

DADOS - Revista de Ciências Sociais, Rio de Janeiro, vol. 60, no-4, 2017, pp. 1095 a 1142. 
da interacción entre las reglas constitucionales, legales e institucionales, y las prácticas organizativas que determinan: (a) las reglas básicas de los procedimientos de la contienda electoral; (b) la organización de campañas, el registro de los votantes y el escrutinio de los votos el día de las elecciones y (c) la resolución de disputas y la certificación de los resultados.

Aún cuando una democracia requiere de elecciones, no acepta cualquier tipo de elecciones (Schedler, 2002; Levitsky y Way, 2004:160). Es más, puede incluso haber elecciones sin democracia (Levitsky y Way, $2004)^{2}$, dado que las elecciones generan legitimidad de origen pero puede que haya reglas, comportamientos y prácticas que convivan con ellas y no sean democráticas. La experiencia reciente evidencia que las elecciones son una condición necesaria pero no suficiente para definir a un sistema político como democrático (Freidenberg, 2013:18) y que, a la vez, puede haber elecciones que convivan con irregularidades, malas prácticas y problemas de gobernanza electoral que condicionan su vitalidad (Donno, 2010; Hyde, 2011; Kelley, 2011; Norris, Frank y Martínez, 2014; Birch, 2015).

A pesar de lo mucho que se ha escrito en las últimas décadas sobre las elecciones, varias preguntas aún no tienen una respuesta satisfactoria en la política comparada. ¿Qué es una buena elección o, desde la perspectiva de la gobernanza electoral, cómo se tienen que hacer buenas elecciones? ¿Cuáles son los atributos necesarios para contar con unas buenas elecciones, más allá de la percepción de los actores, las opiniones de los expertos o de la ciudadanía? ¿En qué medida una elección puede ser técnicamente impecable pero, al mismo tiempo, insatisfactoria para los actores y / o la ciudadanía? Y, finalmente, ¿cómo hacer buenas elecciones en un contexto donde las instituciones de la democracia conviven con malas prácticas o donde se carece de la vigencia del Estado de Derecho? Estas preguntas que aún parecen no tener respuesta no son cuestiones menores. Pensar sobre ellas resulta fundamental no sólo para la academia sino también para los funcionarios electorales que se enfrentan a estos dilemas de manera cotidiana.

Este artículo propone una nueva manera de conceptualizar lo que debe entenderse como una "buena elección". Tras presentar y discutir diversas aproximaciones teóricas (elecciones libres y justas, calidad de las elecciones, malas prácticas, integridad electoral, análisis forense, entre otros) y de evaluar 18 elecciones, se propone definir una "buena 
elección" como aquella que satisface al menos tres condiciones necesarias: a) respeta los estándares internacionales de integridad electoral (procedimiento); b) garantiza el ejercicio de una serie de derechos políticos como la libertad y la justicia; y la ausencia de malas prácticas (contenido) y c) satisface las expectativas de la ciudadanía respecto al modo en que se organizan las elecciones y se consiguen sus objetivos (resultado).

En este estudio exploratorio se analizan, sistematizan y comparan los informes de las Misiones de Observación Electoral de la Organización de los Estados Americanos (OEA) de 18 procesos electorales realizados en América Latina entre 2013 y 2016³. La investigación emplea los informes de las Misiones de Observación Electoral como una herramienta sensible a los aspectos formales e informales (técnicos y simbólicos) de una elección ${ }^{4}$. El uso de los informes para la investigación académica no es nuevo, aunque no han sido empleados de manera extensiva para estudiar las elecciones latinoamericanas ${ }^{5}$.

Las razones que llevaron a elegir esta herramienta para la evaluación de las elecciones son varias. Los observadores han contribuido al conocimiento directo, imparcial y sistemático de los procesos electorales (Carothers, 1997:17; Donno 2010), han generado confianza y legitimidad (Beaulieu y Hyde, 2009:393; Norris, 2014; Merloe, 2015:80) y han ayudado a resolver conflictos intrapartidistas o de los actores políticos con el organismo electoral "detrás de bambalinas" (sin conocimiento de la opinión pública). La credibilidad de los observadores se ha beneficiado además por la inclusión de los principios internacionales de Derechos Humanos para la observación internacional y por el empleo de metodologías sistemáticas comparadas (Merloe, 2015:80), sin el sesgo de la diferenciación que produce el contexto donde se dan las elecciones (Nohlen, 2015:10) o los problemas de contar con información comparable, siempre tan difícil y esquiva de encontrar.

El argumento de este estudio es que, aunque se han incrementado los niveles de calidad de las elecciones en la región en cuanto a dos dimensiones claves (la organización técnica de las elecciones y el respeto a los derechos políticos-electorales), aún existen problemas en cuanto a los resultados (contextos democráticos precarios, bajos niveles de confianza e insatisfacción de la ciudadanía respecto a dichos resultados) y en el contenido (condiciones inequitativas en las que se compite y malas prácticas). El estudio da cuenta en términos teóricos y metodológi- 
cos de las limitaciones del uso de un solo enfoque para el estudio de las elecciones (visión de las elecciones libres y justas, calidad de las elecciones, integridad electoral, análisis forense de las elecciones o malas prácticas) y aboga por una aproximación más holística e integradora, agregando la perspectiva de la calidad de las elecciones, la integridad electoral y la identificación de las malas prácticas, para definir cuando se está ante una buena elección.

El trabajo se estructura en tres partes. Primero, se discuten conceptualmente los diferentes enfoques y herramientas de recolección y análisis de datos de la política comparada y se propone la definición de una buena elección a partir de tomar elementos de tres enfoques (calidad de las elecciones, integridad electoral y malas prácticas). Segundo, se analizan los datos de las elecciones de América Latina en la cuarta maratón electoral (2013-2016), a partir de los informes de las MOE/OEA, y se aplica el "enfoque mixto" para identificar las limitaciones que han experimentado los procesos electorales de la región. Finalmente, se alerta sobre las limitaciones teóricas y empíricas existentes en el estudio y la organización de elecciones en la región y se presentan sugerencias a seguir en esta vigorosa agenda de investigación.

\section{¿QUÉ SON UNAS BUENAS ELECCIONES Y CÓMO EVALUARLAS?: ENFOQUES Y HERRAMIENTAS CONCEPTUALES}

La literatura comparada ha desarrollado diversas aproximaciones teóricas (elecciones libres y justas, calidad de las elecciones, integridad electoral, malas prácticas, análisis forense, entre otros) así como también múltiples herramientas metodológicas y técnicas de análisis cuantitativas y cualitativas para evaluar las elecciones (informes y reportes de los organismos electorales, artículos académicos, la percepción de los expertos y de la ciudadanía, informes y recomendaciones de las misiones de observación electoral y experiencias de observación participante, entre otros). Esto da cuenta de la riqueza de metodologías y evidencia la ausencia de consensos respecto a cómo evaluar las elecciones: cómo definir y cómo medir sus componentes principales para poder delimitar cuando se trata de una buena elección (Cuadro 1).

\section{El Enfoque de las Elecciones Libres y Justas}

Una primera generación de estudios ha respondido a estas preguntas a partir de la idea de que las buenas elecciones son aquellas que cumplen 
dos condiciones: son elecciones libres y justas (Goodwin-Gill, 2005). Avalados por la "Declaración sobre los criterios para elecciones libres y justas", adoptada por unanimidad por el Consejo Interparlamentario en su 154a reunión (París, 26 de marzo de 1994), las elecciones debían cumplir con una serie de requisitos vinculados al ejercicio del voto secreto, la libertad de expresión o la igualdad de las condiciones en el ejercicio de la competencia y el acceso a los medios de comunicación de masas.

Las elecciones son "libres" cuando no hay restricciones normativas para competir, cuando hay libertad para que las candidaturas y sus seguidores puedan movilizarse para conseguir votos y cuando los votantes experimentan poca o ninguna coerción en el ejercicio de su voto. Esto significa que existe una alta dosis de libertad de expresión, movimiento y asociación en la vida política. Las elecciones son "justas" cuando la autoridad es neutral, competente y eficaz contra el fraude y en el conteo de los votos; cuando la policía, el ejército y la corte tratan a los candidatos de manera imparcial; cuando los competidores acceden a los medios y recursos públicos de manera equitativa; cuando los distritos y reglamentos electorales no ponen sistemáticamente en desventaja a la oposición; cuando todos los adultos pueden votar y cuando hay un procedimiento claro e imparcial para resolver reclamos y disputas (Diamond, 2004).

Este paradigma fue el dominante durante mucho tiempo entre los políticos, las organizaciones de observación (OEA, OSCE, UE) y la academia (Goodwin-Gil, 2005; Pastor, 1998) ${ }^{6}$. La legitimidad de una elección se centra en las opiniones de los actores políticos clave y determina que puede ser considerada "libre y justa" si "todos los partidos principales aceptan el proceso y respetan los resultados" (Pastor, 1998: 159). Las críticas a este enfoque tienen que ver con las dificultades para determinar precisamente si una elección es "justa" y "libre". Tradicionalmente, esos criterios eran recogidos en los informes de los observadores internacionales ${ }^{7}$.

\section{El Enfoque de la Calidad de las Elecciones}

Una segunda generación de estudios se ha centrado en evaluar la calidad de las elecciones a partir de indicadores centrados en los "hechos" y no solo en principios normativos (como la legitimidad). Desde este enfoque, se discute en qué medida los comicios son libres y justos 
(como en la perspectiva anterior) pero se incorporan otras dimensiones como la transparencia, la administración de las elecciones o el ejercicio de los derechos de la ciudadanía (Kelley, 2011; Kelley y Kolev, 2010). Se evalúa si las elecciones permiten una competencia equitativa, reflejan lo más fielmente posible la voluntad de los votantes (Mozaffar y Schedler, 2002) y si afectan la calidad de la democracia (Hartlyn y McCoy, 2006; Hartlyn et al., 2008).

Desde esta visión, se valora en qué medida las elecciones cumplen con los "criterios de aceptabilidad" (Hartlyn et al., 2008), que hacen que una elección sea justa y cumpla con los requisitos técnicos, construidos bajo las exigencias de las normas internacionales pero también a partir de juicios de los actores, partidarios y no partidarios; las valoraciones de los medios de comunicación de masas y las opiniones de los observadores nacionales e internacionales (Hartlyn et al., 2008). Diversas investigaciones han desarrollado este enfoque. En sus estudios pioneros, Elklit y Reynolds (2005a; 2005b) se preocupaban por la ausencia de una herramienta que contribuyera a la comparación entre países y procesos y construyeron una medida que evaluara la calidad de las elecciones a partir de más de 50 indicadores. Otros autores han generado bases de datos para medir la "calidad de las elecciones" como la de Duke University (Kelley y Kolev, 2010; Kelley, 2010, 2011) ${ }^{8}$, que permite identificar atributos necesarios (aunque no suficientes) para contar con una elección de calidad'; explorando cómo el tipo de gobernanza electoral existente en el momento de la elección (Hartlyn et al., 2008) o las dificultades del Estado para garantizar la seguridad de la ciudadanía en todo el territorio condiciona la calidad de las elecciones.

\section{El Enfoque de las Elecciones de Integridad}

Las deficiencias del enfoque de las elecciones "libres y justas" así como el de la "calidad de las elecciones" de no poder determinar la calidad del proceso en todo su conjunto (más allá del día de la elección) han buscado ser superadas por otra perspectiva que se centra en la idea de la "integridad de las elecciones" (Norris, 2013; 2014). Si bien no hay un único criterio sobre cómo definir la integridad ni un único grupo de investigación que esté trabajando sobre esta cuestión ${ }^{10}$; la nueva discusión entronca con la idea de contar con elecciones basadas "en los principios democráticos del sufragio universal y la igualdad política [...], caracterizada por una preparación y gestión profesionales, imparciales y transparentes de todo el ciclo electoral" (Norris, 2013:563). 
Esta tercera generación de estudios sostiene que la elección debe ser estudiada como un ciclo (no sólo como un momento que se circunscribe a un día), tal como señalaban originalmente Elklit y Reynolds (2005b) y como postulaban los organismos internacionales ${ }^{11}$. El concepto de "integridad electoral" incluye al menos tres elementos (Norris, 2013; 2014) que deben considerarse en todas las etapas del proceso electoral (pre-electoral, electoral y post-electoral): (i) las elecciones son legítimas si incorporan los valores de las normas globales basadas en acuerdos multilaterales, los convenios internacionales, tratados y leyes internacionales ${ }^{12} \mathrm{y}$, por tanto, respetan normas compartidas que se aplican universalmente a todos los países y tipos de regímenes; (ii) la existencia de malas prácticas, que varían en relevancia de acuerdo con la gravedad de sus consecuencias y (iii) la noción de un ciclo electoral (no limitado a actos fraudulentos el día de las elecciones y sus secuelas).

Este enfoque cambia las herramientas metodológicas, incluye las normas de las convenciones y los estándares internacionales (como hacía el enfoque de elecciones libres y justas) junto a la cuestión de la confianza en las elecciones, a partir de la percepción y juicio de los expertos (Norris et al., 2014; Norris, 2014) y de la ciudadanía (Carreras e Irepoglu, 2013b), empleando encuestas de opinión pública con indicadores comparativos en tiempo y espacio (Birch, 2008; Azpuru, 2015). Con ello se reconoce la brecha entre la ley y la práctica y entre las expectativas y los resultados de las elecciones ${ }^{13}$.

\section{El Enfoque de las Malas Prácticas}

Una cuarta generación de estudios identifica no sólo la integridad del proceso electoral sino, de manera específica, las malas prácticas que hacen que cualquier proceso electoral deje de ser libre, justo, legítimo, equitativo y transparente. Este enfoque no busca establecer parámetros dicotómicos (como los de si hay fraude vs. no fraude o calidad vs. no calidad) sino de tipificar malas prácticas, esto es, "la manipulación del proceso electoral como un asunto privado o personal o conducido por intereses privados, sustituyendo el interés público" (Birch, 2011).

La comprensión de las malas prácticas que integran el menú de formas de manipulación electoral permite profundizar en el conocimiento de lo que son unas buenas elecciones. La literatura académica (Beaulieu y Hyde, 2009; Méndez de Hoyos, 2013) y especialistas de los organismos de monitoreo de las elecciones buscan de este modo sistematizar dife- 
rentes tipos de "malas prácticas". Birch $(2008,2011,2015)$ describe los patrones generales de irregularidades electorales en el mundo así como también los sub-tipos de mala praxis vinculados a la manipulación de las reglas, las condiciones en la que se ejerce la decisión del voto, el mal uso de los recursos del Estado para favorecer a un candidato, el sesgo en la cobertura mediática, la independencia (o no) de la autoridad electoral y los límites a la libre competencia ${ }^{14}$.

Estudios recientes han centrado sus objetivos en el conocimiento de las malas prácticas en las elecciones latinoamericanas (Schedler, 2002; Méndez de Hoyos, 2013; Carreras e Icemoglu, 2013b), dando cuenta que entre otras formas de delito la manipulación del marco legal es la más común en la región (Birch, 2011:48). Sin embargo, poco se sabe aún respecto al modo en que se manipulan esas reglas dentro de la ley, las condiciones en las que éstas se dan o los factores que pueden estar asociados a una mayor manipulación. Si bien este enfoque es aún reciente, contribuye a identificar lo que no está (de lo que está) y favorece una visión más realista de los problemas que condicionan la celebración de las buenas elecciones.

\section{Análisis Forense de las Elecciones}

En la última década, diversos investigadores se han centrado en el "estudio forense de las elecciones" (Leeman y Boschler, 2014; Jiménez e Hidalgo, 2014), a partir de aplicar la teoría de Benford a la detección de fraudes electorales (Mebane, 2006) ${ }^{15}$. La idea de este enfoque, que también centra su atención en la identificación de malas prácticas a través de sofisticadas técnicas estadísticas, es monitorear los valores extremos de los patrones habituales de los resultados electorales y de algunas de las prácticas más comunes como el registro de electores, la participación electoral, los niveles de votos en blanco, los votos nulos, entre otros.

Con la intención de identificar las posibles irregularidades que llevan al fraude de una manera más global, el análisis forense de las elecciones busca una variedad de pistas potenciales que permitan conocer las probabilidades existentes en cada caso de que se lleven a cabo malas conductas (Leeman y Boschler, 2014:34) ${ }^{16}$. Por ejemplo, Jiménez e Hidalgo (2014) descubrieron patrones anómalos en la elección del Referéndum Revocatorio en Venezuela de 2004 y señalaron a dicho proceso como un punto de inflexión en la integridad de las elecciones en el 
¿Qué es una Buena Elección?: El Uso de los Informes de las Misiones...

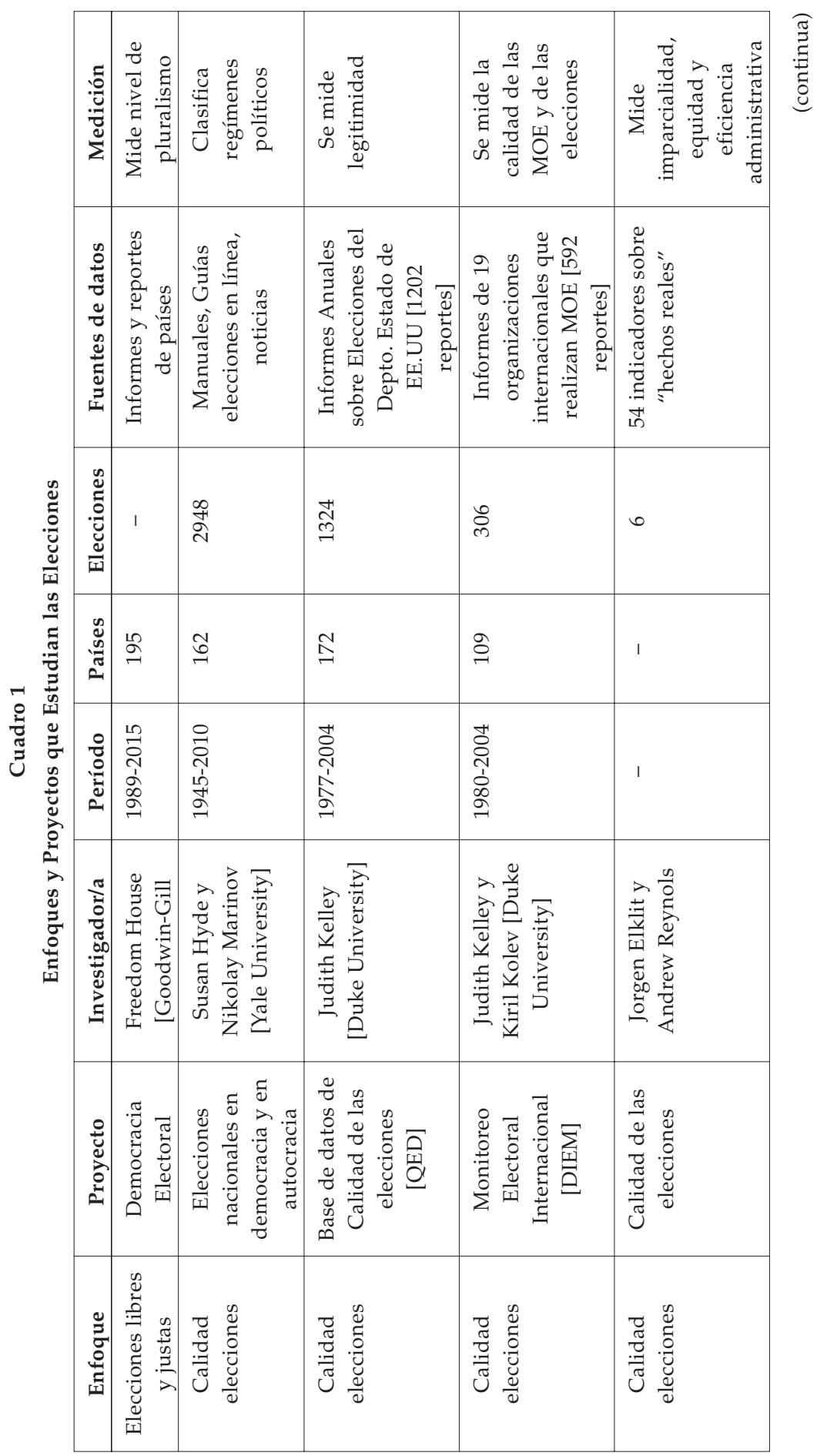

DADOS - Revista de Ciências Sociais, Rio de Janeiro, vol. 60, $\mathrm{n}^{\circ}$ 4, 2017 


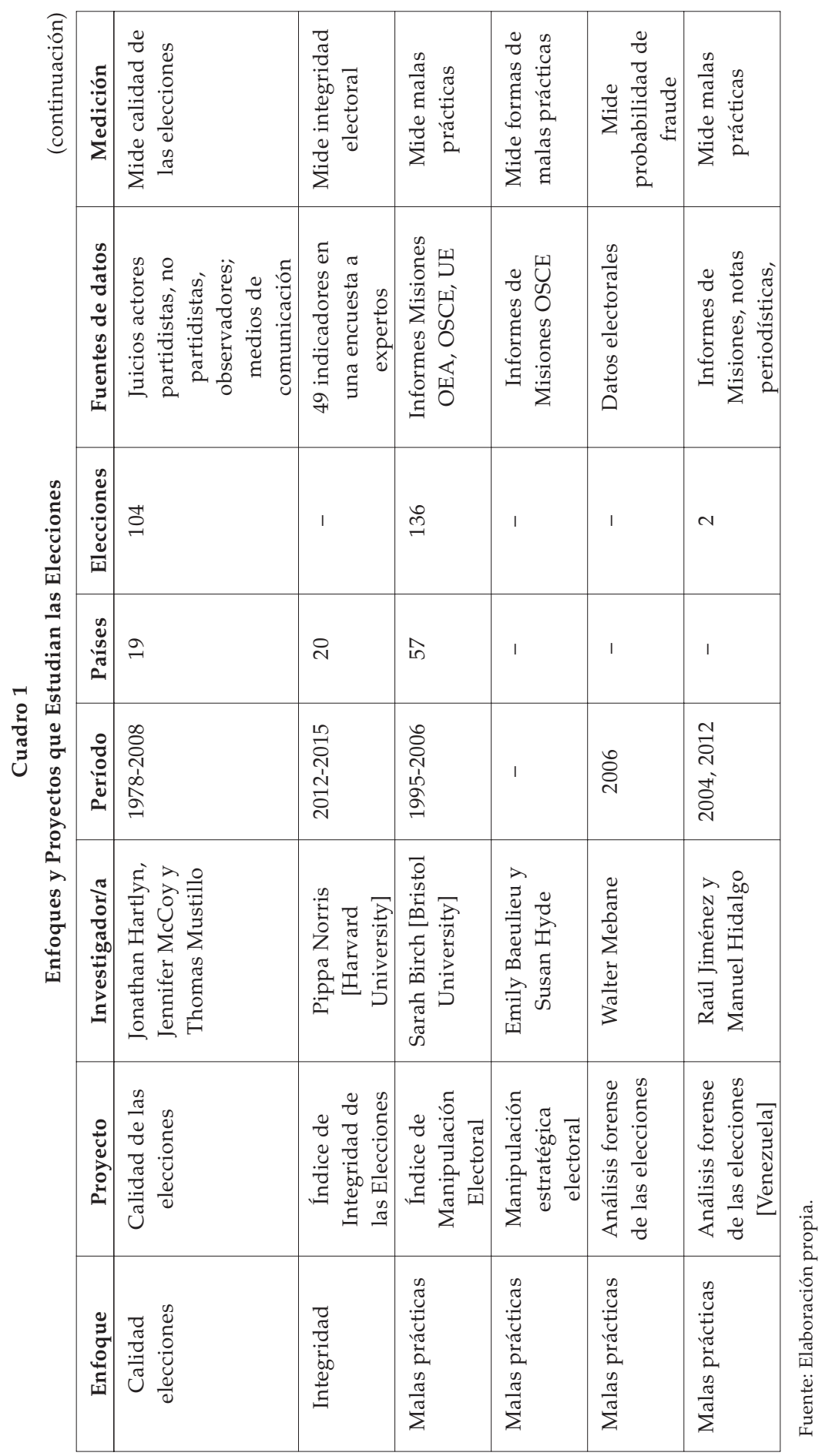

1104 DADOS - Revista de Ciências Sociais, Rio de Janeiro, vol. 60, no 4, 2017 
país. La investigación realizada con técnicas estadísticas sofisticadas puso en duda la fiabilidad del registro electoral en esa elección, a partir de variaciones irregulares en el censo que fueron decisivos para ganar la mayoría del $50 \%$ en el Referéndum y en las elecciones presidenciales de 2012.

Las críticas a este enfoque residen en el uso exclusivo de técnicas cuantitativas para identificar las irregularidades, ya que estas solas no tienen la capacidad de detectar todos los tipos de malas prácticas como las que pueden surgir a partir de análisis más cualitativos como una cobertura sesgada de los medios, la manipulación de las leyes electorales, la distribución inequitativa de los recursos, las prácticas clientelares, las dificultades que enfrentan las mujeres para competir en las elecciones o las restricciones en el registro de partidos, por mencionar algunos.

\section{Enfoque Mixto: Herramientas Conceptuales de la Calidad, la Integridad Electoral y las Malas Prácticas}

Una alternativa a estos enfoques es generar una aproximación que toma elementos de diversas propuestas teóricas, buscando superar las limitaciones presentes en las anteriores. En este enfoque mixto el principal elemento es el de definir las elecciones a partir de la propuesta que hace Morlino (2007) de "calidad de la democracia"17. Se asocia la idea de que una buena elección es aquella que tiene "calidad". De este modo, la calidad se relaciona con 1) los aspectos del procedimiento fijados de manera específica que se debe cumplir para conseguir un determinado producto; 2) el contenido de ese producto, que tenga ciertas características específicas y 3) la satisfacción expresada por el consumidor, independientemente de cuáles sean los procedimientos y sin necesidad de considerar directa y explícitamente los contenidos del producto o servicio o los procedimientos usados para tener ese producto o servicio, sino confiando simplemente en el resultado.

Las tres diferentes nociones de calidad "se formulan en relación a los procedimientos, al contenido y al resultado" (Morlino, 2007) y esta es precisamente la idea que se busca asociar a la definición de lo que es una "buena elección", como aquella que satisface tres condiciones necesarias: a) respeta los estándares, normas y valores internacionales que suponen la integridad electoral (lo que estaría vinculado al procedimiento de cómo organizar una elección); b) garantiza el ejercicio de una serie de derechos políticos vinculados a la libertad y la justicia 
(contenido) y c) satisface las expectativas de la ciudadanía respecto al modo en que se organizan las elecciones y se consiguen sus objetivos sistémicos (resultados).

El segundo elemento tiene que ver con asumir que lo que hay que observar son "hechos" (como señala el enfoque de calidad de las elecciones) y no principios normativos o percepciones de los académicos o de los actores (como señala la integridad electoral). Se trata de identificar "lo que está bien"; en qué medida cumplen con las normas, los valores y exigencias internacionales (como postula el enfoque de la integridad electoral) pero también lo que está ausente, lo que falta y lo que se hace mal (como señala el enfoque de las malas prácticas).

El tercer elemento introduce una idea clave y superadora del enfoque de la integridad electoral y tiene que ver con la idea de ciclo electoral. Las elecciones no pueden ser evaluadas sólo un día, deben ser vistas como un proceso, un ciclo con fases y etapas, centradas en la dinámica de la profesionalización y la transparencia de la gestión electoral. Como sostiene Nohlen (2015:4-5), se toman en cuenta dos dimensiones: a) la visión integral del proceso, que supone la relación entre valores y prácticas y b) la vigencia del Estado de Derecho, la profesionalización de la gestión electoral, la regulación del financiamiento y el acceso a los medios de comunicación (Norris, 2013:563).

A partir de estas premisas, se construye de manera inductiva una matriz centrada en tres dimensiones del concepto de "buena elección" o una "elección de calidad" (resultado, contenido, procedimiento), cada una con una serie de indicadores, a partir de la cual se observan los procesos electorales (Cuadro 2). La dimensión que analiza el resultado de las elecciones trata de identificar el contexto (tipo de régimen político) y la percepción de la ciudadanía en relación a la elección, ya sea en cuanto al nivel de confianza y a la satisfacción con los resultados de dicho proceso $^{18}$. Una elección puede ser impecable en términos técnicos, pero que la ciudadanía no crea que el organismo electoral realiza su tarea con imparcialidad o que piense que se ha realizad o fraude, lo que genera desconfianza en el resultado.

La dimensión que analiza el contenido de las elecciones trata de evaluar en qué medida se respeta el ejercicio de los derechos políticos electorales de la ciudadanía, que se vinculan con la libertad y la justicia, así como también se analizan en qué medida se dan las condiciones de una competencia equitativa. En esta dimensión se evalúan diversos 
¿Qué es una Buena Elección?: El Uso de los Informes de las Misiones...

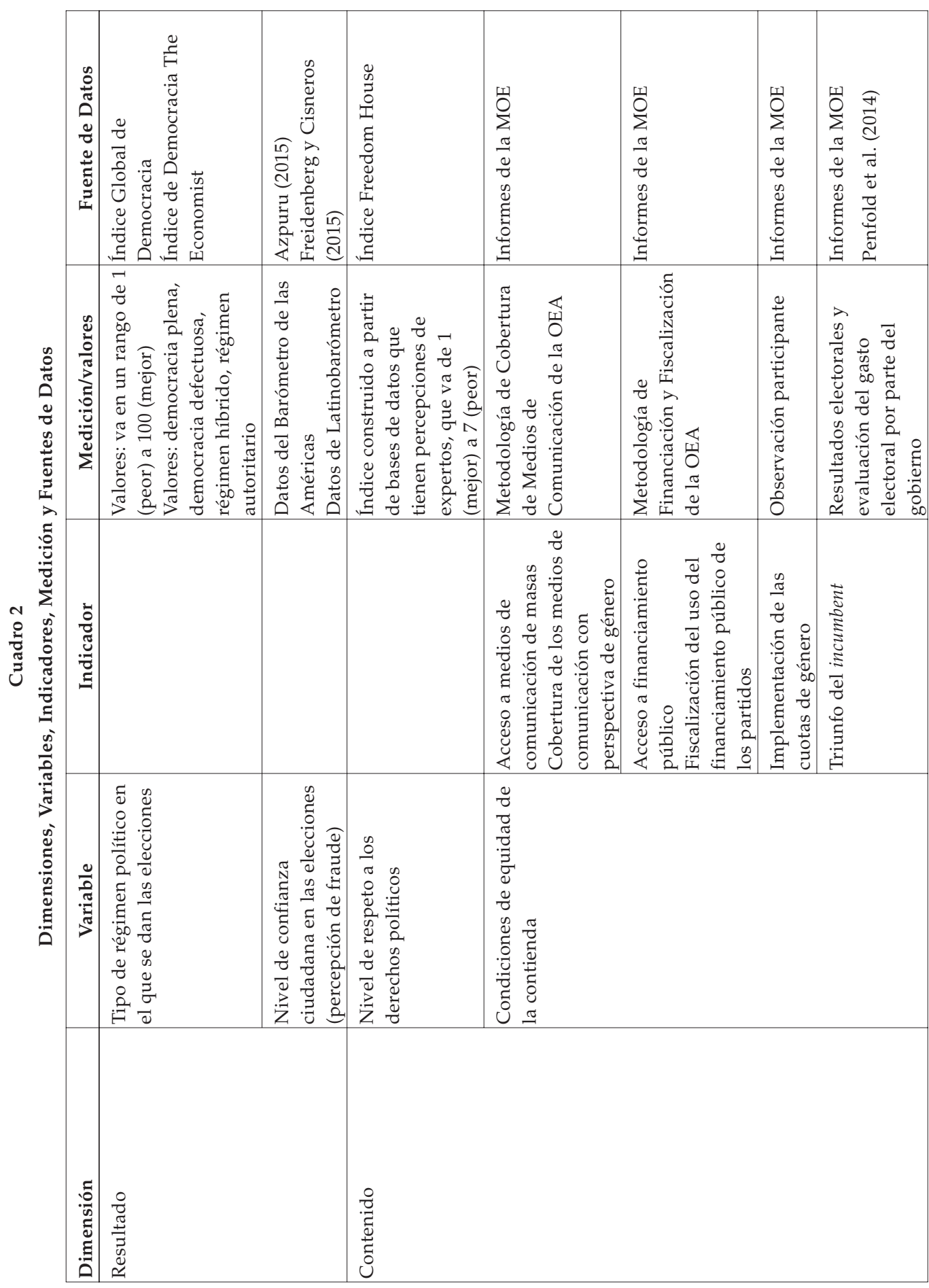

DADOS - Revista de Ciências Sociais, Rio de Janeiro, vol. 60, n 4, $2017 \quad \mathbf{1 1 0 7}$ 


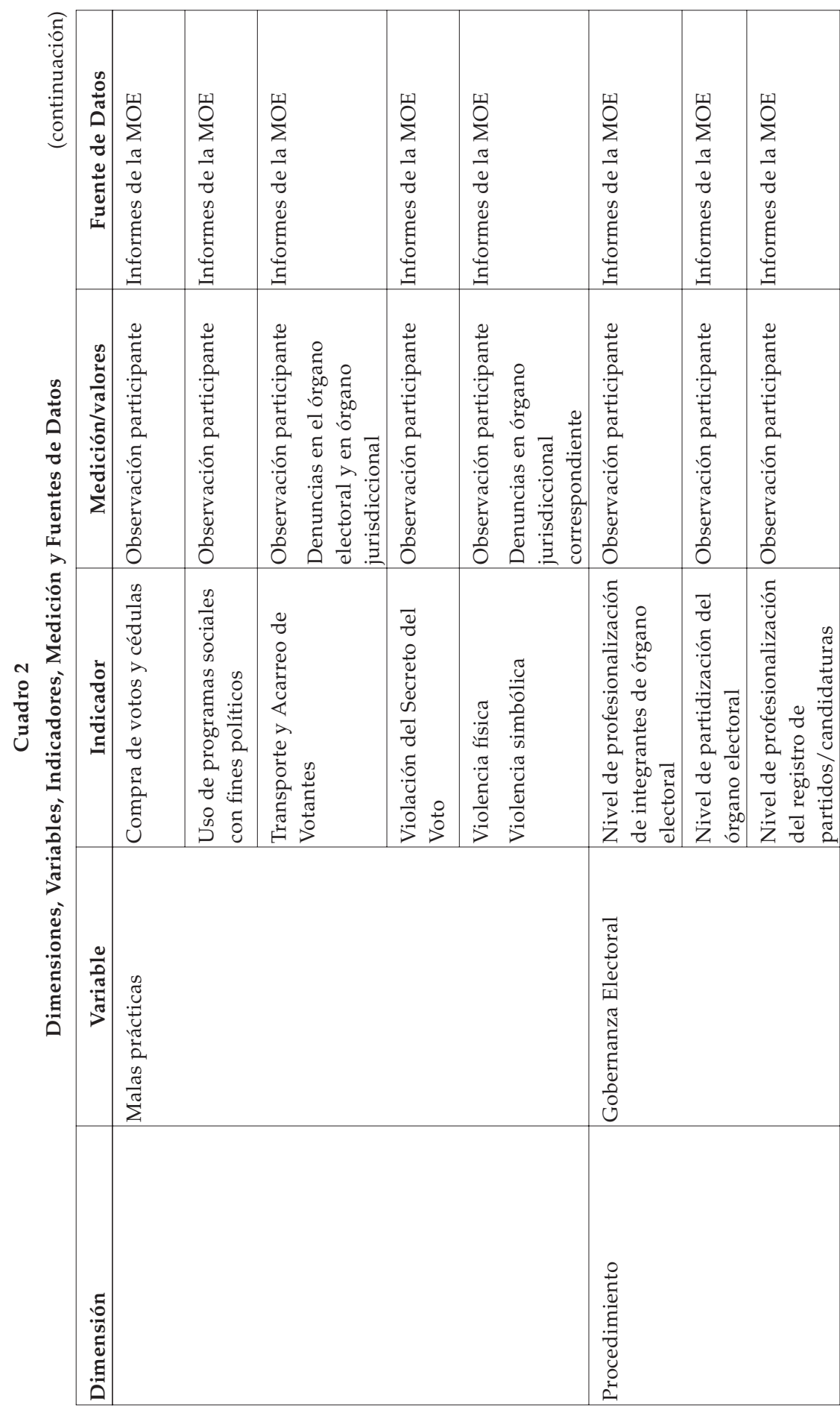

1108 DADOS - Revista de Ciências Sociais, Rio de Janeiro, vol. 60, nº 4, 2017 
¿Qué es una Buena Elección?: El Uso de los Informes de las Misiones...

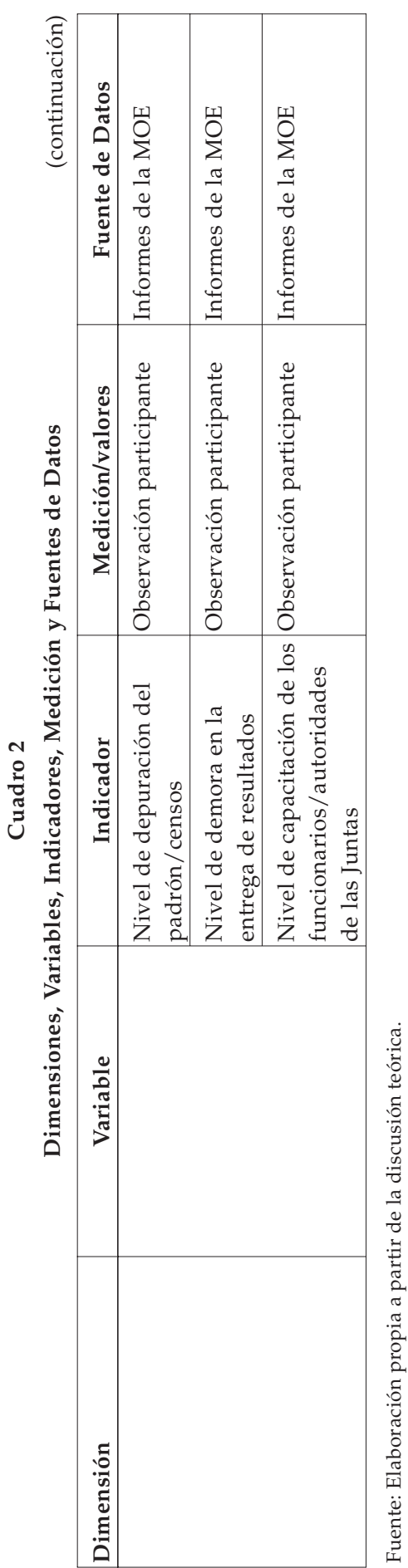

DADOS - Revista de Ciências Sociais, Rio de Janeiro, vol. 60, nํㅜ 4, 2017 
indicadores que tienen que ver con la posibilidad de medir las condiciones de la competencia en relación a los medios de comunicación de masas y la cobertura con perspectiva de género, el acceso a los recursos públicos y la fiscalización de su uso por parte de los partidos, el nivel de respeto a las medidas de acción afirmativa (leyes de cuotas y /o paridad de género) en la configuración de las candidaturas, entre otros. También esta dimensión permite identificar si se llevan a cabo malas prácticas cuando se compite por un cargo de representación popular (movilización clientelar, acarreo, compra de votos, violencia política física y/o simbólica, entre otros).

La dimensión que analiza el procedimiento conoce qué se debe llevar a cabo para organizar las elecciones, esto se vincula a la gobernanza electoral y tiene que ver con el nivel de profesionalización de los órganos electorales, el mecanismo de designación de las autoridades electorales y su vínculo con los partidos políticos (nivel de partidización), la gestión de la organización de las elecciones (logística), la limpieza y nivel de depuración del registro y / o padrón electoral, el nivel de demora en la entrega de los resultados y el nivel de capacitación de los funcionarios/autoridades de las Juntas Receptoras de Voto, entre otros.

En este estudio se evalúan los informes de 18 procesos electorales realizados en América Latina entre 2013 y 2016 (2013: Paraguay, Honduras, El Salvador, Ecuador; 2014: Costa Rica, Panamá, Colombia, Perú, Bolivia; 2015: El Salvador, Bolivia, México, Guatemala, Colombia, Paraguay; 2016: Costa Rica, Perú y Rep. Dominicana). Se hace uso de la información que proveen los Informes de las Misiones de Observación Electoral, con datos tanto cuantitativos como cualitativos, fruto de su observación directa de los procesos electorales. Las ventajas de emplear los informes de la OEA se encuentran en la amplia cobertura de las elecciones de la cuarta maratón electoral (2013-2016), el acceso a la información (pueden consultarse todos los informes de manera on line), el análisis de todo el ciclo electoral (y no sólo el día de la elección), contando con expertos por temas; el alto nivel de profesionalización que han alcanzado en el proceso de elaboración y redacción de los informes (tienen una estructura similar, permiten identificar datos para medir indicadores comunes, siguiendo una misma metodología), lo que facilita la comparación entre elecciones de un mismo país en diferentes momentos y en elecciones de diversos países.

Si bien algunas investigaciones señalan desventajas del uso de los informes ${ }^{19}$, lo cierto es que las organizaciones internacionales se han ido 
profesionalizando cada vez más, generando una fuente valiosa de información sistematizada, no se concentra en el día de la elección sino que tiene varias fases y equipos en diferentes etapas del ciclo, generando una amplia cobertura para la mayoría de las elecciones realizadas y generan recomendaciones, que son públicas, y que están acompañadas de asistencia técnica tanto en tiempo electoral como no electoral a petición de cada país ${ }^{20}$.

\section{LOS DATOS: LAS ELECCIONES EN AMÉRICA LATINA A LA LUZ DE LOS INFORMES DE LAS MOE/OEA}

Durante el ciclo 2013-2016, América Latina ha vivido un intenso calendario electoral, ya que en ese período se han celebrado unas 23 elecciones (Cuadro 3). Esta no es la primera vez que la región vive un intenso período electoral. En otras tres oportunidades (1989, 1994, 2005-2006), también un número significativo de países tuvieron elecciones generales concurrentes (presidenciales, legislativas y / o municipales), lo que da cuenta de la existencia de un ciclo de elecciones cada determinado tiempo que se ha denominado "maratones electorales" (Zovatto, 2006).

De las 23 elecciones que se realizaron en la cuarta maratón electoral, este artículo analiza 18 procesos electorales, no pudiendo incluir aquellos casos en los que las MOE/OEA no han sido invitadas a observar (como ocurre en Venezuela, Argentina, Chile, Uruguay y Brasil). Los informes de las MOE/OEA proveen información que se sistematiza en torno a tres dimensiones, en relación a los resultados, los contenidos y los procedimientos de las elecciones.

\section{Resultados: Tipo de Régimen Político, Nivel de Confianza y Satisfacción de la Ciudadanía en las Elecciones}

La mayoría de los países de la región ha celebrado sus elecciones de la cuarta maratón electoral en un escenario poliárquico con dinámicas de competencia estables, que han permeado una serie de prácticas, formales e informales, con al menos mínimas condiciones de equidad, libertad y justicia en el territorio; cierta alternancia entre las opciones políticas e incluso incorporando a sectores sociales que se sentían excluidos en los procesos electorales (como las mujeres, los indígenas, los afros, entre otros). 


\section{Flavia Freidenberg}

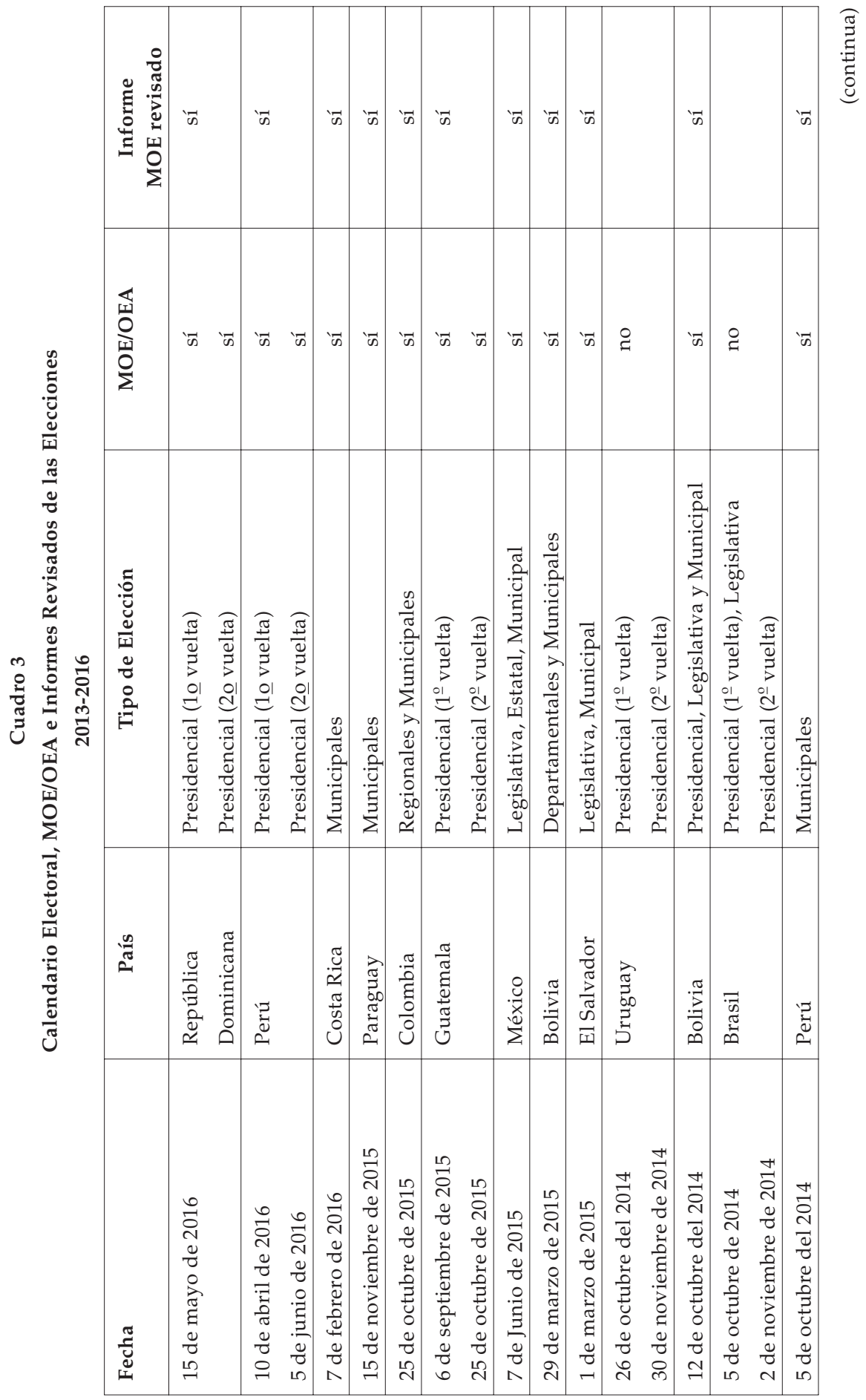

1112 DADOS - Revista de Ciências Sociais, Rio de Janeiro, vol. 60, no 4, 2017 
¿Qué es una Buena Elección?: El Uso de los Informes de las Misiones...

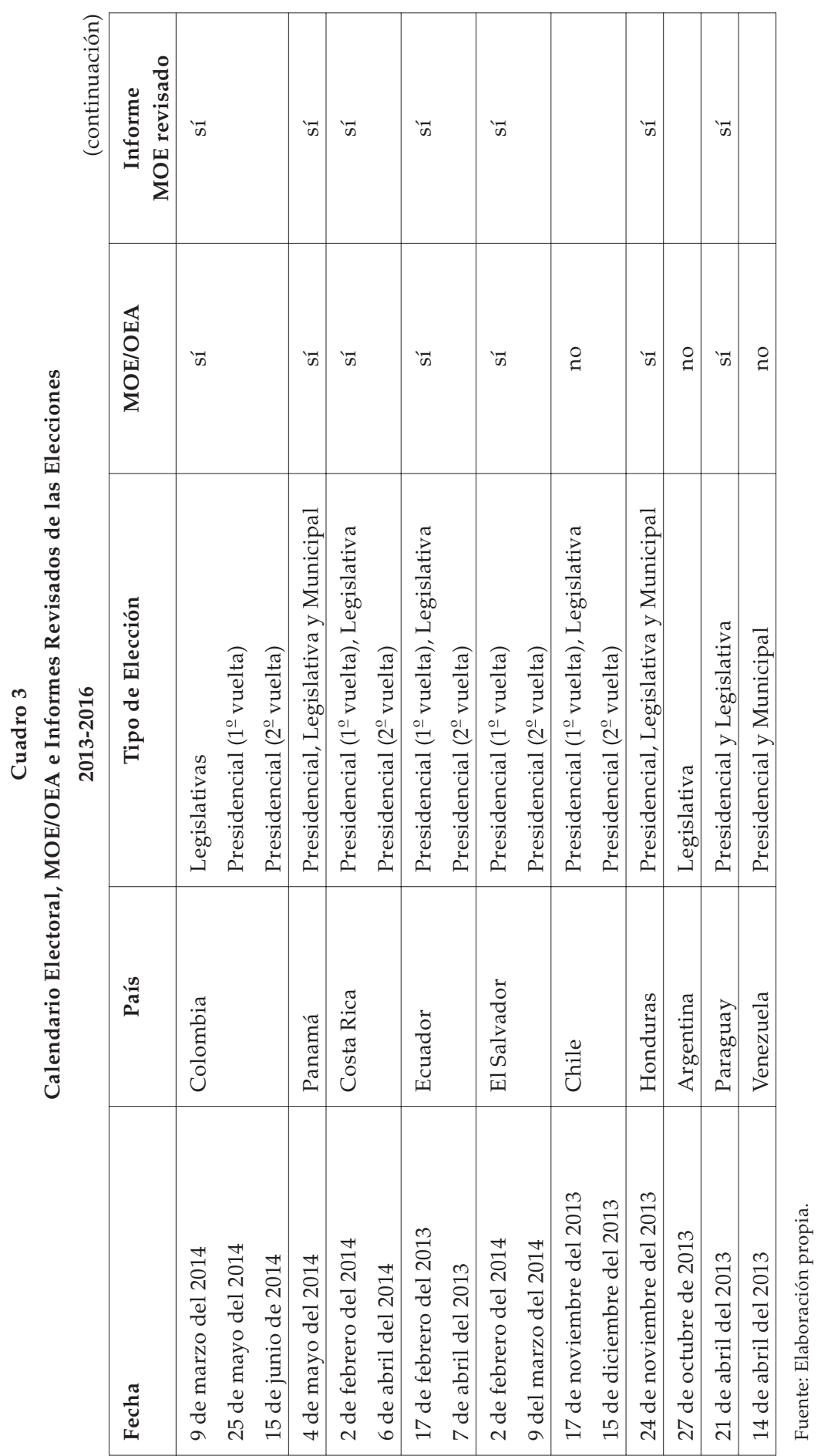


Los Informes MOE/OEA confirman que las elecciones cumplieron con los parámetros de la perspectiva de las "elecciones libres y justas", realizando procesos de manera periódica, posibilitando la alternancia política y con ciertos niveles de competencia entre los contendientes, a pesar de las diferencias en el tipo de régimen político vigente en el que se celebraron las elecciones (Tabla 1). Esta no es una cuestión menor. Las condiciones del entorno en el que se celebran las elecciones son fundamentales para contar con buenas elecciones (Nohlen, 2015:4-5), sobre todo cuando se trata de un régimen híbrido (Ecuador, Bolivia, Honduras, Guatemala) o una democracia defectuosa (Colombia, Paraguay, México) (Azpuru, 2015) ${ }^{21}$.

Al mismo tiempo, las elecciones cuentan cada vez con menos confianza de la ciudadanía (Freidenberg y Cisneros, 2016; Azpuru, 2015), aunque esto varía por países. El Latinobarómetro 2015 muestra la opinión que tiene la ciudadanía sobre las elecciones en sus países (Gráfico 1$)^{22}$. En Chile y Uruguay, la percepción sobre lo fraudulentas que son las elecciones apenas alcanza porcentajes de 18 y 12 por ciento, lo cual da cuenta de que su ciudadanía aún confía en sus instituciones y en los actores políticos que participan en dichos procesos. A diferencia de ello, en México y Brasil, la percepción que tiene la ciudadanía sobre las elecciones es mayoritariamente negativa. En México, el 67 por ciento opina que las elecciones son fraudulentas, mientras que en Brasil lo hace el 58

\section{Gráfico 1}

Percepción sobre las Elecciones en América Latina

Fraudulentas/Muy Fraudulentas

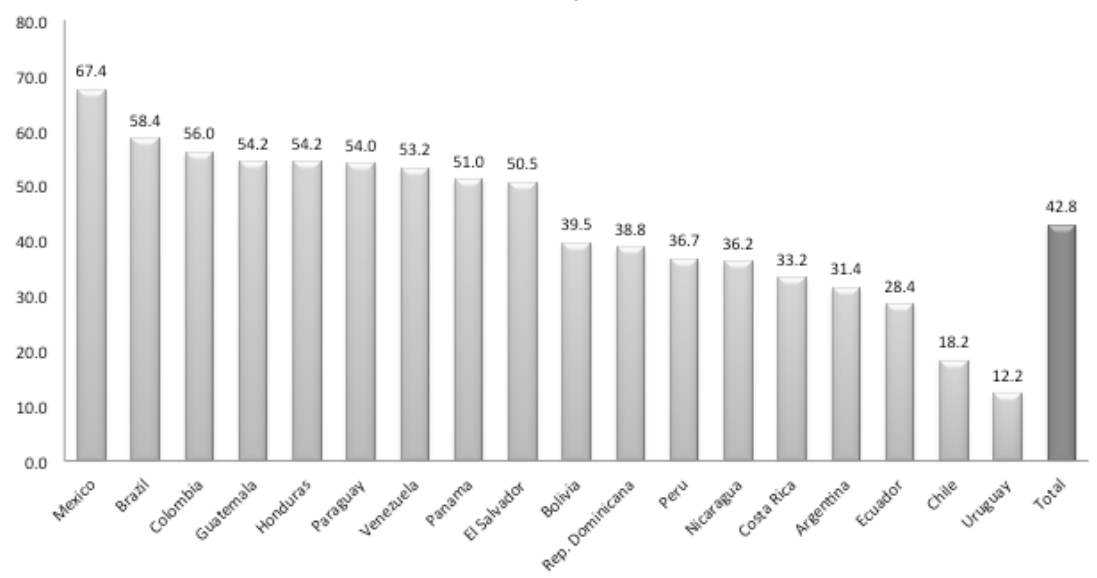

Fuente: Elaboración a partir del Latinobarómetro (2015) y publicado previamente en Freidenberg y Cisneros (2016). 
¿Qué es una Buena Elección?: El Uso de los Informes de las Misiones...

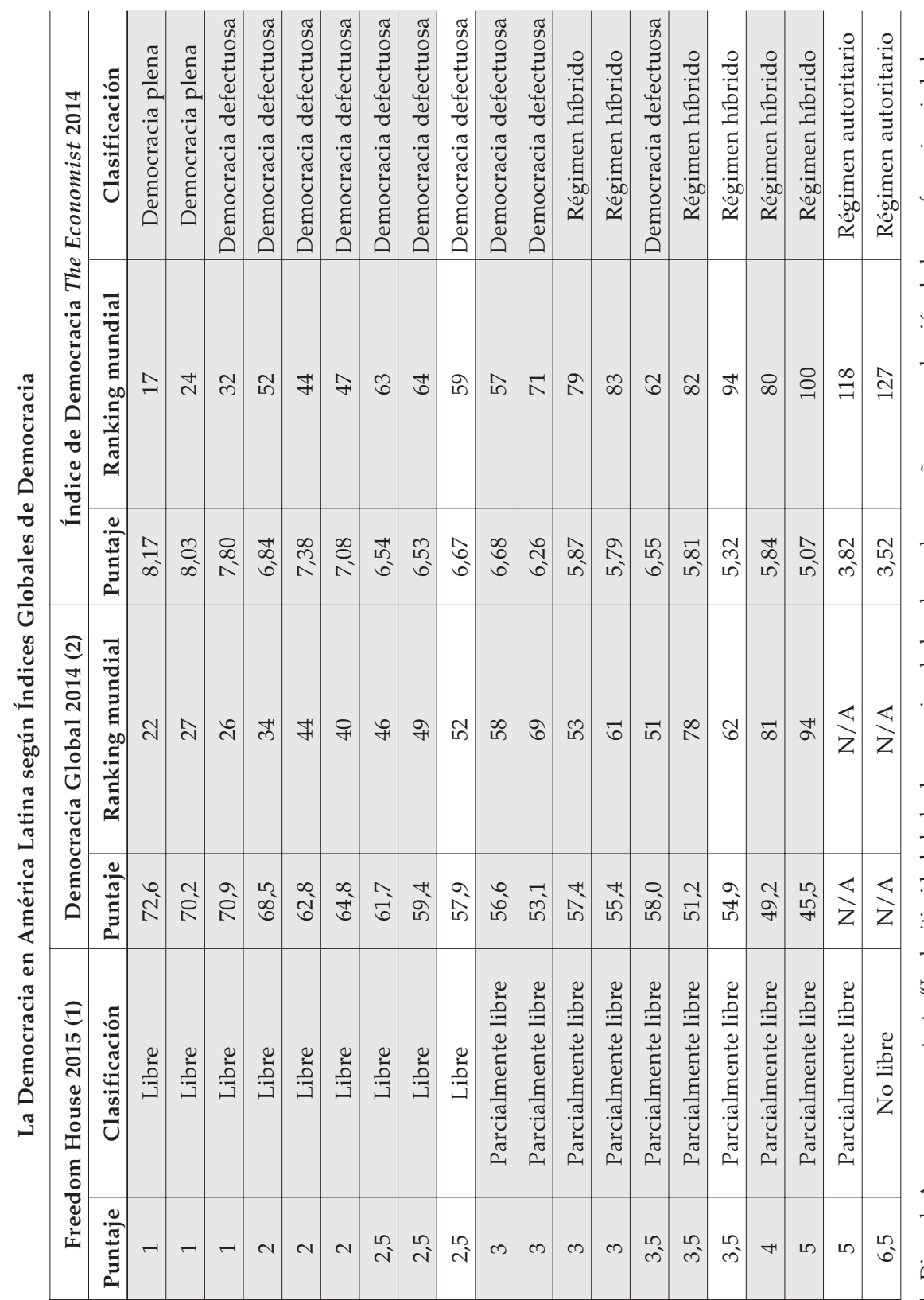

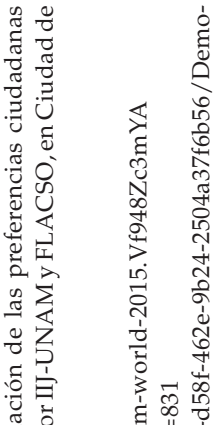

o.

这古

준. 가

के के

客究

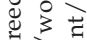

进

ปั

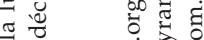

政

월

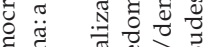

ฮิ

ส ฮ

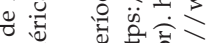

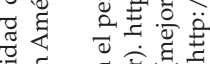

घี चี

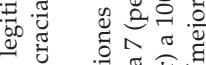

ฮ

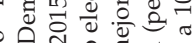

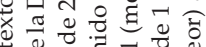

क

๘

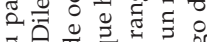

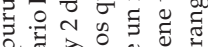

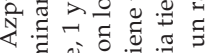
ᄃ कू

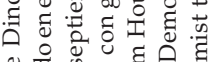

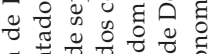

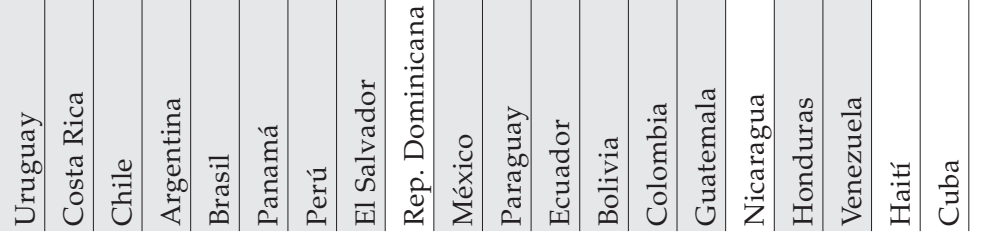

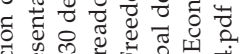

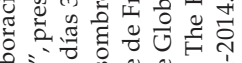

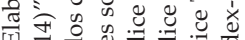

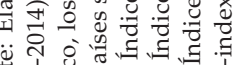

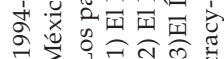


por ciento. Entre estos extremos, se encuentra una gran cantidad de países ubicados mayoritariamente en torno a la media regional, que alcanza el 43 por ciento.

Asimismo, la insatisfacción con la democracia se encuentra asociada positivamente con la percepción de fraude en las elecciones, esto significa que a medida que se incrementa la insatisfacción con el desempeño del sistema democrático aumenta la percepción de que las elecciones son fraudulentas, pues la correlación, que se mide de 0 a 1 , alcanza un puntaje alto de 0.8 (Freidenberg y Cisneros, 2016). Los casos con mayor grado de insatisfacción con la democracia, como México, tienen una mayor percepción de fraude en los comicios y, de manera opuesta, donde el nivel de insatisfacción con la democracia apenas alcanza el 30 por ciento, como en Uruguay, la percepción también es baja (Gráfico 2).

Finalmente, la mayoría de los sistemas políticos donde se han celebrado elecciones 2013-2016 han tenido dificultades para garantizar el ejercicio del monopolio legítimo de la coacción física en todo el territorio (como en México, Brasil, Honduras, Guatemala, Argentina o El Salvador); erradicar la presencia de grupos paraestatales que controlan o manipulan al Estado (paramilitares, mafias o crimen organizado) y minimizar el poder de caudillos locales que permean y limitan las institu-

\section{Gráfico 2}

Percepción de Fraude en Elecciones e Insatisfacción con la

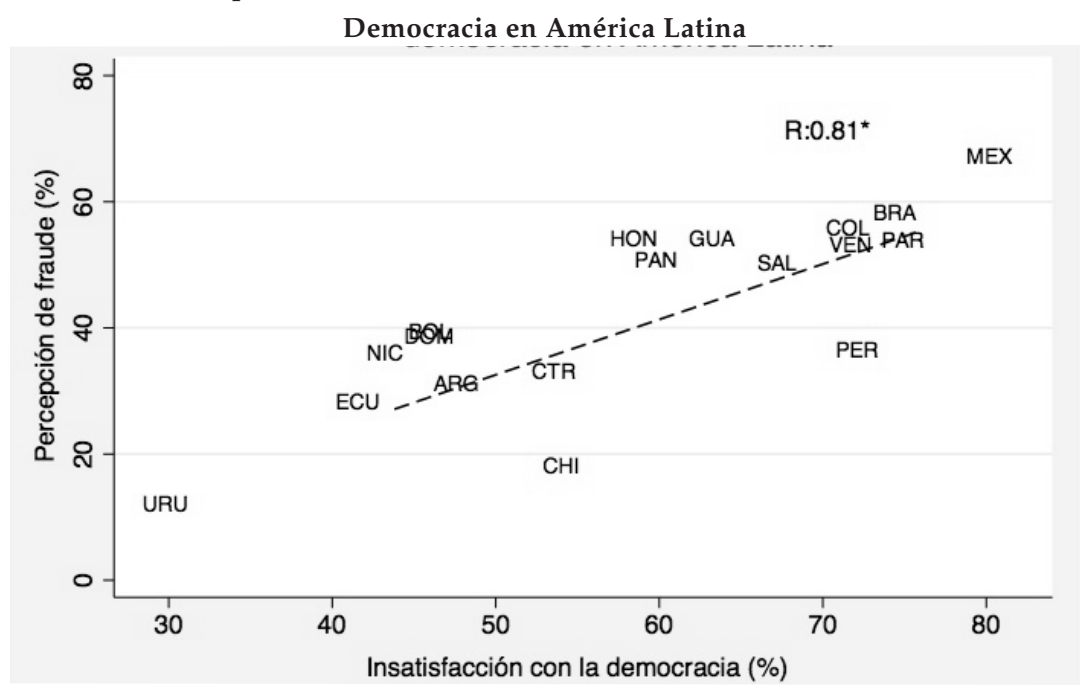

Fuente: Elaboración propia con datos de latinobarómetro 2015. * Significativo estastíticamente. 
ciones poliárquicas a nivel subnacional (Freidenberg y Suárez-Cao, 2014).

Muchos de los órganos electorales han podido hacer elecciones técnicamente impecables pero algunos de esos sistemas políticos no pueden garantizar la vigencia del Estado de Derecho. Por ejemplo, la elección mexicana del 7 de junio de 2015 enseñó las dificultades de hacer procesos electorales cuando el Estado no puede asegurar la libre circulación de los electores (Oaxaca, Chiapas, Michoacán); garantizar la seguridad de los candidatos (con cerca de 20 muertos); identificar, sancionar y eliminar la compra de votos o garantizar la distribución del material electoral en los diferentes distritos durante la jornada electoral (Chiapas o Oaxaca).

\section{Contenido: Nivel de Respeto a los Derechos Políticos, Condiciones Inequitativas para la Competencia y Malas Prácticas}

Las elecciones 2013-2016 presentaron dificultades en las condiciones de equidad de la contienda, por la "manipulación estratégica de las elecciones" (Beaulieu y Hyde, 2009) así como también por la existencia de diversos tipos de malas prácticas e irregularidades (Beaulieu y Hyde, 2009; Méndez de Hoyos, 2013), sin que necesariamente sean formas de fraude. Las condiciones de la competencia en varios de los países han sido inequitativas, en lo que se denomina de "cancha inclinada", como lo muestran los datos de los informes de las MOE/OEA y la investigación de Penfold et al. (2014) (Tabla 2).

Salvo en dos elecciones, en el resto de los procesos el candidato en el gobierno triunfó siempre en la búsqueda de su reelección ${ }^{23}$. Los incumbents que ganan elecciones de manera reiterada no necesariamente son una potencial fuente de riesgo para la democracia. El problema está cuando controlan los recursos del Estado en favor de su candidatura y eso hace que las elecciones tengan una situación de desbalance entre las diferentes opciones (Birch, 2015), afectando las condiciones de equidad de la competencia y con ello las oportunidades de los contendientes.

En los procesos presidenciales evaluados, en cinco países, se reeligió al incumbent (como evidencia la Tabla 2), dando cuenta de mayores probabilidades de que se diera "cancha inclinada" por el uso de recursos del estado a favor del candidato de gobierno. En Colombia, tras perder en la primera vuelta, finalmente Santos consiguió la reelección conse- 
cutiva en un escenario altamente polarizado. Lo mismo ocurrió en Ecuador, donde Correa ganó la reelección con más de 30 puntos porcentuales de diferencia frente al segundo candidato. En Venezuela, Maduro garantizó el proyecto de la revolución chavista, en un contexto de alta polarización y gran competitividad contra la oposición de la Mesa de Unidad. En Bolivia y Brasil también se reeligió al presidente o al partido que estaba en el poder, garantizando la continuidad del proyecto gubernamental.

Tabla 2

Los Incumbents siempre ganan en las Elecciones Presidenciales de América Latina

\begin{tabular}{|c|c|c|c|c|c|c|}
\hline & ¿ $1^{0} \stackrel{0}{0} 2^{0} ?$ & $\begin{array}{c}\text { Ganador } \\
\%\end{array}$ & $\begin{array}{c}\text { Segundo } \\
\%\end{array}$ & $\begin{array}{c}\text { MV } \\
\text { 1을 Vuelta }^{\circ}\end{array}$ & $\mid \begin{array}{c}\text { MV } \\
2^{\circ} \text { Vuelta }\end{array}$ & Ganador/a \\
\hline Venezuela 2013 & $1^{\mathrm{o}}$ & 50.61 & 49,12 & 1.49 & & Incumbent \\
\hline Costa Rica 2014 & $2^{-}$ & 77.22 & 22,1 & & 55.6 & $\begin{array}{c}\text { Partido } \\
\text { Desafiante }\end{array}$ \\
\hline El Salvador 2014 & $2^{\underline{o}}$ & 50.11 & 49.89 & & 0.22 & $\begin{array}{c}\text { Partido } \\
\text { Incumbent }\end{array}$ \\
\hline Colombia 2014 & $2^{-}$ & 50.95 & 45.00 & & 5.95 & Incumbent \\
\hline Panamá 2014 & - & 39.1 & 31.4 & 7.7 & & $\begin{array}{c}\text { Partido } \\
\text { Desafiante }\end{array}$ \\
\hline Honduras 2013 & - & 36.89 & 28.78 & 8.11 & & $\begin{array}{c}\text { Partido } \\
\text { Incumbent }\end{array}$ \\
\hline Paraguay 2013 & - & 45.83 & 37.11 & 8.72 & & $\begin{array}{c}\text { Partido } \\
\text { Desafiante }\end{array}$ \\
\hline Ecuador 2013 & $1^{\mathrm{o}}$ & 57.17 & 22.68 & 34.49 & & Incumbent \\
\hline Chile 2013 & $2^{\underline{o}}$ & 62.17 & 37.87 & & 24.34 & $\begin{array}{c}\text { Partido } \\
\text { Desafiante }\end{array}$ \\
\hline Brasil 2014 & $2^{\underline{o}}$ & 51.63 & 48.37 & 8.04 & 3.26 & Incumbent \\
\hline Bolivia 2014 & $1^{\mathrm{o}}$ & 61.04 & 24.49 & 36.55 & & Incumbent \\
\hline Uruguay 2014 & $2^{\underline{o}}$ & 56.62 & 43.38 & 16.93 & 13.24 & $\begin{array}{l}\text { Partido } \\
\text { Incumbent }\end{array}$ \\
\hline
\end{tabular}

Fuente: Elaboración propia a partir de datos electorales oficiales.

A pesar de que hubo competencia entre diferentes partidos, la inequidad se puso de manifiesto en el acceso al financiamiento electoral como señalan los informes de las Misiones de Observación Electoral [iMVE Colombia 2014; iMOE Panamá 2014; iMOE Honduras 2013; iMOE Paraguay 2013 y 2015; iMOE Perú 2014; iMOE Costa Rica 2016; iMOE Dominicana 2016]. También en las dificultades para implementar la fiscalización [iMVE México 2015; iMOE/OEA Honduras 2013; 
iMVE Colombia 2014 y 2015; iMOE/OEA Bolivia 2014; iMOE Panamá 2014]; en el bajo nivel de rendición de cuentas por parte de los partidos [iMOE Honduras 2013; iMOE Paraguay 2013 y 2015; iMOE El Salvador 2015], su escasa profesionalización [iMOE Costa Rica 2016; iMOE Paraguay 2015] o en la complejidad de un sistema de financiación que no resulta transparente ni equitativo [iMOE Honduras 2013; iMOE Dominicana 2016] (Cuadro 4).

La inequidad se expresó en el bajo nivel de sanción por incumplir la cuota de género en las candidaturas [iMOE Honduras 2013; iMOE Paraguay 2013; iMVE Colombia 2014; iMOE Perú 2016]; en que los partidos continúen colocando a las candidatas mujeres, a los afros o a los indígenas en "distritos perdedores", es decir, aquellos donde no van a ganar [iMOE Costa Rica 2016; iMOE Perú 2014; iMOE Panamá 2014; iMVE 2014; iMOE Paraguay 2013] ${ }^{24}$ o en las dificultades de cobertura de las mujeres candidatas frente a los hombres [iMVE México 2015; iMOE Panamá 2014; iMOE Honduras 2013; iMOE Paraguay 2013; iMOE Costa Rica 2014 y 2016]. ${ }^{25}$ Es más, en el caso de Paraguay, la iMOE/OEA 2015 ha sugerido la incorporación de una cuota de género en las listas de candidaturas a los cargos de elección popular, dado que es uno de los países que carece de este tipo de mecanismo de acción afirmativa en la actualidad (junto a Guatemala y Panamá).

Las elecciones pusieron en evidencia el acceso desigual de los partidos y / o candidaturas al dinero público [iMOE Paraguay 2013; iMOE Costa Rica 2014 y 2016; iMOE Ecuador 2014 / 2013; iMOE Bolivia 2014, iMOE Perú 2014; iMOE El Salvador 2015; iMOE Dominicana 2016]; a los medios de comunicación de masas [como en Costa Rica iMOE 2014/2016; iMOE El Salvador 2013/2015; iMOE Perú 2014; iMOE Colombia 2014; iMOE Panamá 2014; iMOE Ecuador 2014, por mencionar algunos]; las mayores oportunidades de los que están en el poder en el acceso a los medios de comunicación [iMOE Ecuador 2014; iMOE Bolivia 2014; iMOE Paraguay 2013]; en el abuso de la publicidad ilegal [iMOE Honduras 2013; iMOE Colombia 2014], muchas veces oculta detrás de la propaganda gubernamental (iMOE Bolivia 2014) y en el desarrollo de campañas anticipadas por los partidos y sus candidaturas [iMOE Ecuador 2013; iMOE Bolivia 2014; iMOE Paraguay 2013] (Cuadro 4).

La política requiere dinero (Casas Zamora y Zovatto 2015). En algunos países, a pesar de estar regulado, no se hace efectiva la entrega de dinero del financiamiento público a los partidos por parte del Estado 


\section{Flavia Freidenberg}

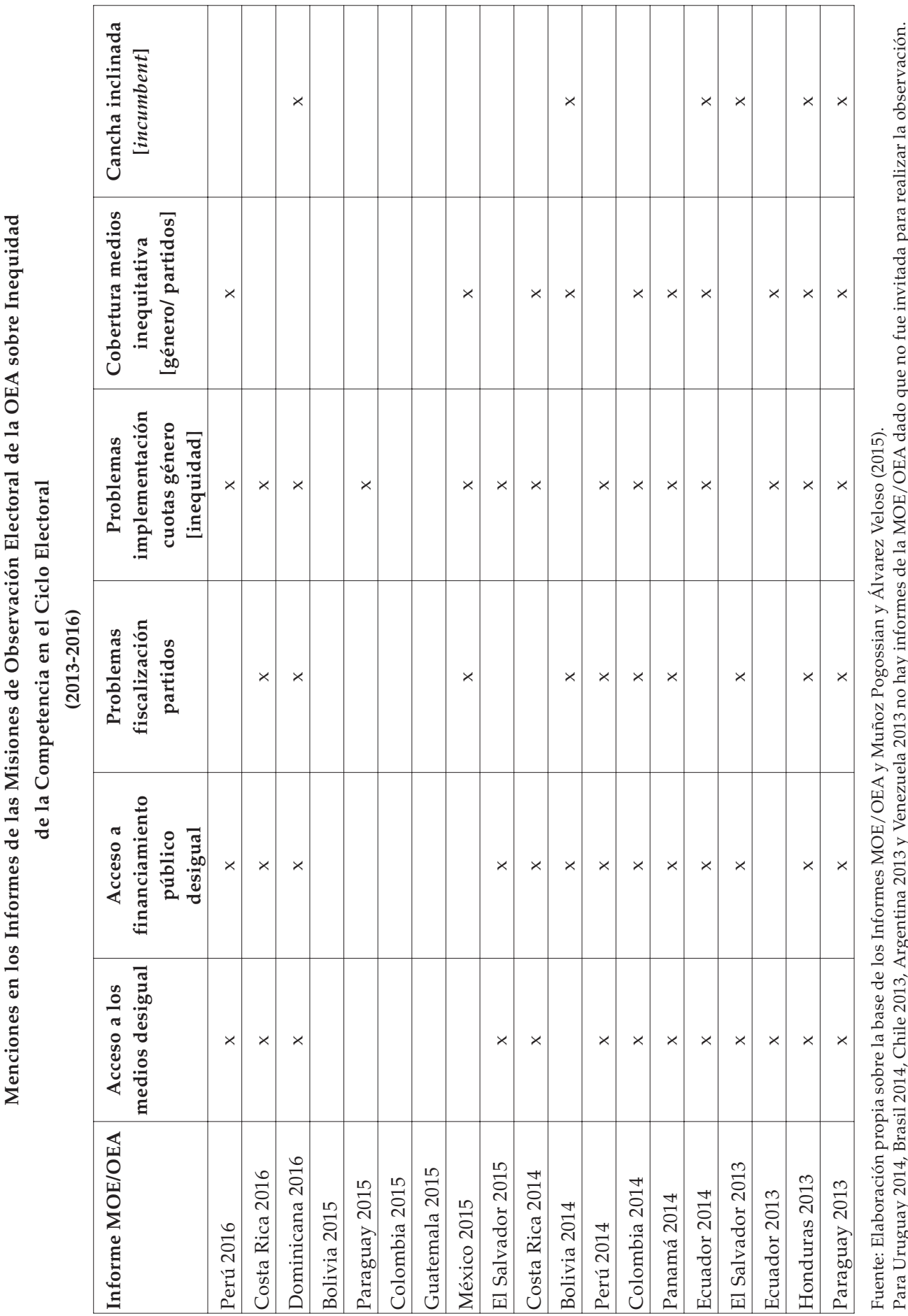

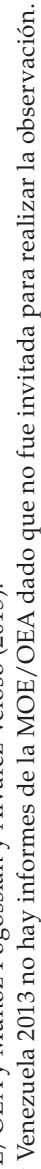

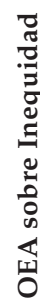

1120 DADOS - Revista de Ciências Sociais, Rio de Janeiro, vol. 60, no 4, 2017 
[iMOE Perú 2014 y 2016], no hay financiamiento público en época no electoral [iMVE Colombia 2014], las franjas electorales en los medios son escasas o pequeñas [iMOE Panamá 2014] o los partidos no destinan el dinero que por ley se les obliga a la capacitación y fortalecimiento de la participación de las mujeres [iMVE Colombia 2014; iMOE Costa Rica 2014; iMOE Panamá 2014; iMOE Costa Rica 2016]²6.

Los informes destacaron además la necesidad de fiscalizar los recursos que se les da a los partidos. No se trata sólo de darles financiamiento para sus actividades (algo que en Perú no está ocurriendo aún) sino de regular las donaciones y, fundamentalmente, el tope de gastos por cargo público y de fiscalizar en qué gastan los recursos públicos [iMOE El Salvador 2015; iMOE Costa Rica 2014; iMOE Panamá 2014; iMOE Paraguay 2013 y 2015; iMVE Colombia 2015] ${ }^{27}$. En Costa Rica, tanto en 2014 como en 2016, además, se planteó la dificultad que tienen los partidos para acceder a financiamiento por adelantado al proceso electoral [Costa Rica iMOE 2014; iMOE 2016], lo que limita su acceso a una contienda equitativa.

Diferentes tipos de malas prácticas están todavía presentes en las elecciones latinoamericanas (Méndez de Hoyos, 2013). En el período 2013-2016 esto no cambió. Los informes dan cuenta de denuncias sobre acarreo de votantes, vinculados a la compra de voto [iMVE Colombia 2014 y 2015; iMOE Paraguay 2013]; de la violación del secreto del voto [iMOE Honduras 2013; iMOE Colombia 2014 y 2015; iMOE Paraguay 2013], de candidatos que ganan elecciones desde la cárcel [iMOE Perú 2014] y de violencia física [iMOE Dominicana 2016], incluso por el acoso hacia mujeres candidatas [iMOE Bolivia 2014; iMVE México 2015], muerte de periodistas realizando su trabajo [iMVE México 2015] o de miembros de la fuerza pública [iMOE Perú 2014] (Cuadro 5). En las entidades estatales mexicanas además hubo intimidación, descrédito y violencia contra las candidaturas (en particular, contra las mujeres), lo que se consideran malas prácticas, algo que sería muy difícil de observar si solo se analizaran las elecciones desde el enfoque de "las elecciones libres y justas" (iMVE México 2015).

\section{Procedimiento: Gobernanza Electoral}

La gestión de las elecciones debe ser eficaz, eficiente, transparente y autónoma tanto del gobierno como de los partidos en general. El papel protagónico de los órganos de la gobernanza electoral tiende a ser más 
Flavia Freidenberg

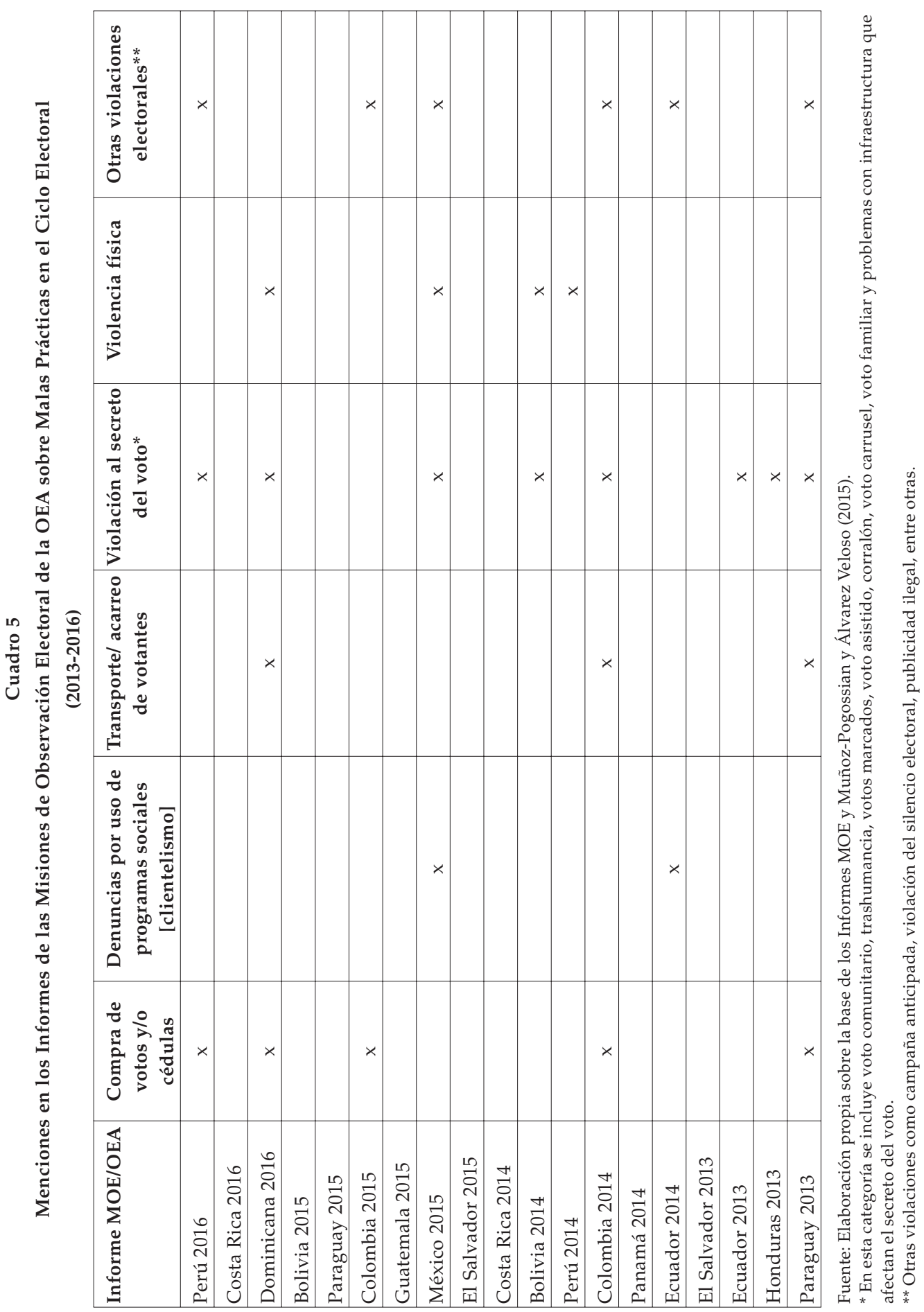

1122 DADOS - Revista de Ciências Sociais, Rio de Janeiro, vol. 60, no 4, 2017 
visible en las democracias emergentes o en aquellas en las que son débiles los procesos administrativos y altos los niveles de desconfianza entre los actores políticos (Hartlyn et al., 2008). Cuanto más independientes sean los órganos de la gobernanza electoral de los partidos, mejores serán las condiciones para realizar "buenas elecciones". Por el contrario, cuando las instituciones electorales responden a intereses partidistas pueden ser menos capaces de realizar su función principal: producir certidumbre sobre a los resultados y los procedimientos empleados en la elección.

Los Informes alertaron sobre la precariedad de la gobernanza electoral en varios de los países donde se llevaron a cabo elecciones y sobre la necesidad de una mayor profesionalización de los organismos electorales [iMOE Honduras 2013; iMOE El Salvador 2013 y 2015; iMOE Ecuador 2014], debido a sus bajos niveles de ciudadanización, su temporalidad (sólo se instalan en tiempo de elecciones) [iMOE El Salvador 2015; iMVE Colombia 2014], los problemas que se generan por la ausencia de un servicio civil profesional electoral [iMOE Honduras 2013] y por la partidización de los nombramientos de las instituciones electorales [iMOE Honduras 2013, iMVE Colombia 2014 y iMOE El Salvador 2015].

También se registraron problemas en las gestiones y la logística de las elecciones en la región. La OEA solicitó que se cambie el procedimiento de entrega de listados de miembros para la composición de las mesas de escrutinio, lo que no se adecua a los estándares internacionales [iMOE Honduras 2013; iMOE Costa Rica 2016]; que se mejore la capacitación de los miembros de las mesas [iMOE Honduras 2013; iMOE Perú 2014; iMOE Bolivia 2014 y 2015; iMOE Costa Rica 2014; iMOE Ecuador 2014; iMVE Colombia 2014] y de las casillas [iMVE México 2015]; la depuración de los censos y padrones electorales [iMOE Honduras 2013; iMOE Bolivia 2014 y 2015; iMOE El Salvador 2015; iMVE Colombia 2015; iMOE Paraguay 2015], que se hagan más eficientes los tiempos de instalación de las casillas [iMVE México 2015] y de las JRV [iMOE Costa Rica 2016; iMOE Perú 2016]; en la entrega y transmisión de los resultados [iMOE Honduras 2013; iMOE Bolivia 2014 y 2015; iMOE Perú 2014; iMVE México 2015]; en la transmisión de los resultados preliminares [iMOE Bolivia 2014 y 2015] y en las dificultades en las condiciones de acceso de la ciudadanía con discapacidad a los centros de votación [iMOE Bolivia 2015]. 
Las MOE/OEA también identificaron problemas en las condiciones de trabajo de los organismos electorales, debido a la sobrecarga administrativa en materia de gobernanza electoral y de fiscalización a los partidos [iMVE México 2015]; la falta de coordinación entre los órganos electorales y la des-armonización legislativa de los calendarios electorales [iMVE México 2015] ${ }^{28}$, los criterios que se emplean para conformar las Juntas Receptoras de Votos [iMOE Costa Rica 2016; iMOE Paraguay 2015], la debilidad de las fiscalías [iMVE México 2015; iMOE Panamá 2014]; la necesidad de sistemas de medios de impugnación integrales [iMOE Dominicana 2016] y la falta de concientización frente a los delitos electorales por parte de la ciudadanía (iMVE Colombia 2014 y 2015) (Cuadro 6).

La OEA llamó la atención respecto a la ausencia de representantes de partidos como miembros de las Juntas Receptoras de Votos [iMOE Costa Rica 2016], aún cuando el órgano electoral ha podido poner en marcha el mecanismo alternativo de la figura de "auxiliar electoral" así como también de cierta confusión entre los miembros de las Juntas Receptoras de Voto sobre cómo realizar la apertura de las mesas hasta el proceso de escrutinio [iMOE Costa Rica 2016; iMOE Bolivia 2015; iMOE Paraguay 2015; iMOE Dominicana 2016]; de impuntualidad en la instalación de las mesas [iMOE Perú 2016; iMOE Dominicana 2016] y de que dichas mesas estuvieran integradas con muy pocos miembros titulares, la mayoría de ellos suplentes, generando espacio para que los apoderados de los partidos asumieran funciones que correspondían a los miembros de mesa [iMOE Paraguay 2015; iMOE Perú 2016; iMOE Dominicana 2016].

\section{CONCLUSIONES PRELIMINARES Y LOS RETOS DE UNA AGENDA DE INVESTIGACIÓN EN CONSTANTE CRECIMIENTO}

La ciudadanía tiene derecho a que las elecciones sean auténticas y genuinas (Merloe, 2015:81) y, por ello, a tener buenas elecciones. En esta investigación se propuso entender a las "buenas elecciones" como aquellas que, respetando los estándares internacionales, cumplen con los criterios de calidad en el procedimiento (método), en el contenido (atributos) y en el resultado (percepción), sin el ejercicio de malas prácticas que condicionen su dinámica y funcionamiento. Las buenas elecciones generan democracias de calidad, que se desarrollan en contextos de institucionalidad estatal fuerte; donde las condiciones de supervivencia de la ciudadanía están aseguradas; las reglas son imparciales, 
¿Qué es una Buena Elección?: El Uso de los Informes de las Misiones...

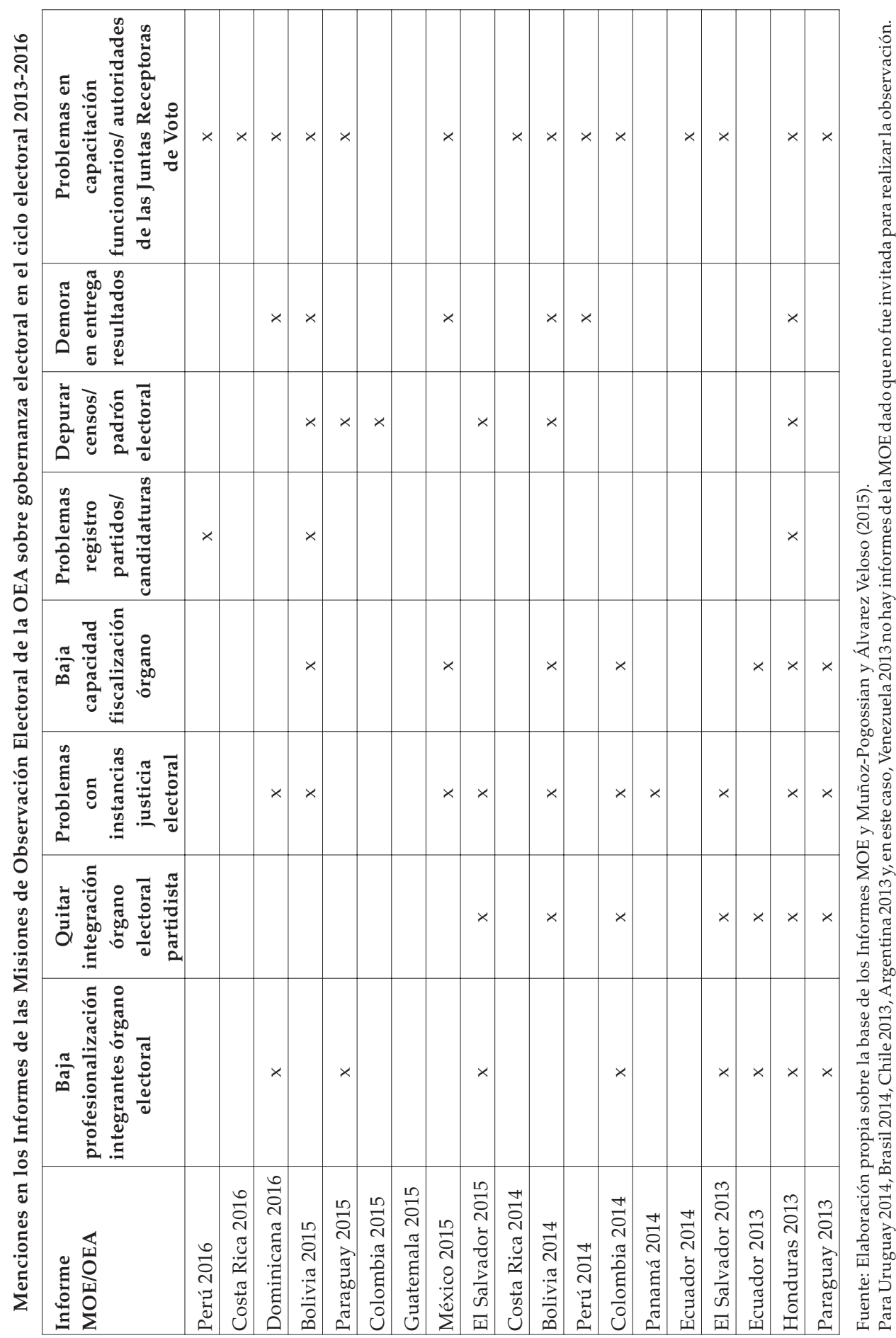

DADOS - Revista de Ciências Sociais, Rio de Janeiro, vol. 60, nº 4, 2017 
conocidas, valoradas y respetadas por todos; los organismos electorales gozan de legitimidad; el Estado de Derecho está extendido y se persiguen, castigan o impiden las malas prácticas y todas las opciones partidistas y las élites y los ciudadanos aceptan los resultados y están satisfechos con el desarrollo de esas elecciones.

Los procesos electorales pueden estar acompañados de eventos extraordinarios como crisis institucionales, presidentes destituidos, escándalos de corrupción, violencia política, movilización social, denuncias de fraude, desconfianza ciudadana e incluso pueden suponer algún margen de irregularidades menor (Nohlen, 2015:5; Elklit y Reynolds, 2005a:150). Es más, pueden ser técnicamente perfectos pero que las élites no acepten los resultados o que la ciudadanía, descontenta con el funcionamiento de la democracia, desconfíe o rechace el modo en que se llevó a cabo la elección. La tensión entre hechos y expectativas aún no está resuelta y depende de la fortaleza de las instituciones y la cultura política de la ciudadanía.

El conjunto de indicadores que definen unas buenas elecciones funcionan como un "tipo ideal", dada la dificultad de que las elecciones sean perfectas. Si bien las deficiencias electorales son heterogéneas y su nivel de gravedad varía en los distintos países que celebran elecciones (y en un mismo país en el tiempo), sus consecuencias para la legitimidad del sistema democrático son muy preocupantes (Norris, 2014). Desde escenarios de represión explícita y directa a partidos políticos de oposición, la compra de votos y las redes clientelares hasta problemas en el padrón electoral, las condiciones de cancha inclinada de la competencia o la partidización de la función electoral, son algunas muestras de los déficits en la calidad electoral y con ello de la calidad de la democracia.

A pesar de las ilusiones y las declaraciones (siempre) optimistas de los que hacen las elecciones y de los que las ganan, las elecciones perfectas no existen. En este sentido, las elecciones perfectas son más bien una aspiración conceptual que tienen en mente quienes las estudian, las organizan, las observan y / o las califican. Aún cuando la política comparada ha hecho esfuerzos significativos por mejorar las herramientas para estudiar las elecciones (que pueden evidenciarse en los diversos enfoques que se han presentado en este estudio), aún se requiere mayor trabajo sistemático, de corte inductivo, que permita llenar un vacío conceptual respecto a lo que es una buena elección y cuáles son los indicadores que permitan definirlo. Esta es una cuestión importan- 
te no solo para los académicos, también los funcionarios electorales, que son quienes las organizan y los electoral practitioners, que deben evaluar y calificar elecciones desde las organizaciones internacionales o las agencias de cooperación. En este sentido, este trabajo buscó aportar en la identificación de los mejores indicadores y las más óptimas herramientas para calificar unas buenas elecciones.

La evaluación de la experiencia de la cuarta maratón electoral en América Latina contribuye en esta tarea y da cuenta de que aún cuando las elecciones estén rutinizadas en los países de la región como "eventos cotidianos y rutinarios", esto no está exento de problemas que limitan las condiciones de la competencia y de malas prácticas que afectan el modo en que se garantizan los derechos y se ejerce la democracia. Esta investigación da cuenta de cómo los informes de las Misiones de Observación contribuyen en la identificación de los factores que condicionan la realización de buenas elecciones.

Las MOE/OEA de 2013-2016 han evidenciado diversos problemas que condicionan las elecciones y, con ello, la calidad de la democracia. Primero, la ausencia de un contexto socioeconómico, institucional y político propicio para desarrollar buenas elecciones. Aún cuando los organismos electorales no son responsables directos de cómo opera ese contexto, resulta difícil evaluar las elecciones sin tener en cuenta las condiciones del entorno en las que se celebran, las peculiaridades institucionales y las características socioeconómicas de los votantes. Segundo, las condiciones de inequidad de la competencia, tanto por la cobertura sesgada de los medios como por el acceso inequitativo a los recursos económicos y las condiciones de cancha inclinada que favorecen a las candidaturas en el poder. Tercero, la existencia de malas prácticas (como el clientelismo, la compra de votos, las encerronas, violación del secreto del voto, entre otras) y, cuarto, los problemas técnicos en la organización electoral y las dinámicas de la gobernanza electoral. Los informes de la OEA alertan sobre la "manipulación estratégica de las elecciones", las violaciones al ejercicio del voto secreto, los problemas de inequidad de la contienda, los bajos niveles de autonomía, alta partidización y baja profesionalización de algunos órganos electorales que funcionan en la región.

Este trabajo reivindica el uso de los informes de las Misiones de Observación Electoral como una herramienta idónea para conocer lo que ocurre en las elecciones. Los informes de las MOE/OEA son sólo un 
instrumento pero definitivamente son una fuente directa, técnicamente responsable y profesional que contribuye a identificar lo bueno y lo malo de cada proceso electoral. Aún así, ellos no son los responsables directos de controlar las elecciones. Otros actores nacionales, como los partidos de oposición, los medios de comunicación de masas e incluso la propia sociedad civil organizada, a través de ONG'S o movimientos sociales, son claves en el ejercicio de control de las elecciones.

La observación internacional puede contribuir en la asistencia técnica de los organismos electorales, amparadas en las reglas y normas internacionales, a partir de realizar recomendaciones (que no son vinculantes) pero que pueden contribuir a identificar áreas de oportunidad para generar cambios que contribuyan a incrementar la calidad de las democracias latinoamericanas. Por ejemplo, los informes para Honduras 2013, Paraguay 2013, El Salvador 2013 y 2015 y Bolivia 2014 destacaron la necesidad de quitar el poder a los partidos en la organización de las elecciones y de separar su organización de las cuestiones jurisdiccionales ${ }^{29}$.

También han recomendado la profesionalización de los organismos electorales [iMOE Honduras 2013]; la depuración y actualización del padrón electoral [iMOE Paraguay 2015; iMVE Colombia 2015]; la capacitación de la ciudadanía que participa en el proceso como autoridades de las mesas [iMOE Bolivia 2015; iMOE Paraguay 2015] y la profesionalización de los partidos políticos [iMOE Costa Rica 2016]. Las Misiones sugirieron campañas de capacitación y formación de nuevos liderazgos al interior de las estructuras partidarias [iMOE Costa Rica 2016; iMOE Paraguay 2015], el uso del financiamiento público indirecto a través de la franja electoral gratuita a los medios de comunicación [iMOE Costa Rica 2016], mejores sistemas de transparencia sobre el origen y el destino de los recursos de los partidos [iMOE Paraguay 2015], la representación más igualitaria de las mujeres [iMOE Costa Rica 2016; iMOE Paraguay 2015; iMOE Perú 2016], en relación a las candidaturas [iMOE Costa Rica 2016] como al interior de las organizaciones de partidos [iMOE Costa Rica 2016; iMOE Dominicana 2016].

Las Misiones recomendaron fortalecer a los partidos políticos como ejes centrales de la competencia. Para ello, sugirieron que los partidos debían estar obligados a mantener estructuras de administración de recursos profesionalizadas con procesos estandarizados, que no dependan de las dirigencias ni que se den sólo en época de campaña 
electoral [iMOE Costa Rica 2016]. También recomendaron hacer simulacros del día de la elección [iMOE Paraguay 2015; iMOE Dominicana 2016]; evitar la trashumancia y la compra de votos [iMVE Colombia 2015].

La evaluación de los informes dan cuenta de la necesidad de que las capacidades desarrolladas por las organizaciones internacionales sean complementarias a las actividades que cada país genera en materia de organización electoral y, en particular, en la observación desarrollada por la ciudadanía. Una tarea importante que realizan las Misiones es hacer transferencia de "tecnología electoral", esto es, de conocimientos, habilidades y buenas prácticas, tanto a los organismos electorales nacionales como a las organizaciones de la sociedad civil que realizan procesos de observación electoral. Con este tipo de tareas se desarrollan capacidades y habilidades locales en materia de observación doméstica, lo que también supone un espacio de participación y de mayor educación cívica para cada país, lo que de manera indirecta puede contribuir en la calidad de la democracia.

La agenda de investigación sobre la evaluación de las elecciones es dinámica. La misma debe incorporar la evaluación de las reglas, de los valores y de las prácticas que se desarrollan en torno a las elecciones. En futuros estudios se deberá evaluar en qué medida las recomendaciones realizadas por las MOE/OEA, o los informes de otras organizaciones internacionales, son incorporadas por las autoridades electorales de cara a contribuir con la calidad de las elecciones. Muchas de esas recomendaciones incluyeron además propuestas de reforma electoral para los países [iMOE Perú 2016; iMOE Bolivia 2015; iMOE Honduras 2013; iMOE Paraguay 2015; iMVE Colombia 2015; iMOE Dominicana 2016], lo que puede convertirse en una oportunidad de cambio de las malas prácticas y los incentivos normativos que las promueven.

Esta investigación concluye que la evaluación del impacto de las recomendaciones aún está por ser realizada en los países de la región así como también resulta necesario desarrollar una metodología sistemática que permita la comparación de las recomendaciones en el tiempo, entre elecciones de un mismo país, y entre países. De nada sirve que se lleven a cabo dichas recomendaciones, si éstas finalmente no se toman en cuenta, no son públicas, no son socializadas con la ciudadanía ni incluidas como mejoras para la realización de las próximas elecciones en el país donde se celebró la observación. Los mecanismos de evaluación 
respecto a la inclusión de las recomendaciones en la normativa de los países están por ser definidos y discutidos y deben ser revisados en la siguiente Misión de Observación.

Resulta evidente que las elecciones en América Latina enfrentan al menos tres retos: el desarrollo de capacidad estatal y la vigencia del Estado de Derecho; una mayor equidad en las condiciones de la contienda y un mayor profesionalización de los órganos electorales y de los partidos políticos. No todas estas tareas son responsabilidad del mundo electoral ni mucho menos de las Misiones de Observación Electoral pero sí lo son de las sociedades democráticas. Se trata de procurar y/o de recuperar la confianza de la ciudadanía en las elecciones y con ello en el sistema democrático. No sirve tener elecciones técnicamente perfectas sin Estado ni ciudadanía que pueda elegir libremente. Y esto sí es una tarea de todos quienes pretendan vivir en democracia.

(Recebido para publicação em 1/1/2016) (Reapresentado em 12/2/2017) (Aprovado para publicação em 14/6/2017) 


\section{NOTAS}

1. La poliarquía según Dahl (1971) se caracteriza por la celebración de elecciones competitivas; alto nivel de pluralismo; incertidumbre resultados; todos los candidatos tienen iguales oportunidades de ganar elección; libre expresión de las preferencias política; ausencia de limitaciones en la presentación de las candidaturas y garantías de que va a haber debates plurales y abiertos sobre cuestiones de fondo.

2. La diferencia entre una democracia y un autoritarismo electoral está en que en las democracias las elecciones deben ser competitivas, libres y justas, contar con alguna dosis de incertidumbre, que los partidos pierdan elecciones y que se cumpla los principios de la integridad electoral (Norris, 2014). El punto de quiebre entre una democracia electoral y un autoritarismo electoral está en que las elecciones en una democracia deben respetar unos mínimos de libertad y justicia mientras que en los autoritarismos electorales no.

3. Entre los organismos que realizan observación electoral internacional se encuentran la Organización de Naciones Unidas (ONU), la Organización de los Estados Americanos (OEA), la Unión Europea (UE) o la Organización de Seguridad y Cooperación de Europea (OSCE); asociaciones de parlamentarios (como ParlAmerica); organizaciones no gubernamentales internacionales (como el Centro Carter, European Centre for Electoral Support o el National Democratic Institute) u organizaciones que realizan observación doméstica (como Poder Ciudadano en Argentina; Participación Ciudadana en República Dominicana, SUMA en Venezuela o la MOE en Colombia). En UNASUR, las Misiones no se denominan de "observación" sino "de acompañamiento", son a solicitud del país anfitrión y participan los miembros de los organismos electorales. En las misiones de IIDH-CAPEL suelen participar miembros de los organismos electorales reunidos en UNIORE, el país sede es el que paga la misión y es el que emite las invitaciones a los observadores. No todos los países invitan a la OEA a observar elecciones, dado que Chile, Argentina, Uruguay y Brasil no aceptan la observación internacional.

4. Las Misiones de la OEA reciben otro nombre en tres países: en México, se las denomina "Misiones de Visitantes Extranjeros", en Nicaragua, "Misiones de acompañamiento electoral" y en Colombia, "Veeduría Electoral". En los tres casos, la OEA firma protocolos para equiparar las tareas que realiza con las de la observación (que permiten la libre circulación, la presentación pública de los informes o la posibilidad de hacer declaraciones públicas). A los efectos de esta investigación, se homologará para todos los casos la precisión de MOE cuando se cite a los informes.

5. Diversos proyectos han discutido el uso de esta metodología para profundizar el conocimiento de los procesos electorales, tanto en relación a su calidad, integridad o malas prácticas (Birch, 2008, 2011; Kelley y Kolev, 2010; Merloe, 2015; Melián y Tuccinardi, 2015; Martínez i Coma et al., 2015) e incluso llamando la atención sobre la necesidad de dejar de evaluar elecciones "libres y justas" para realizar "informes más holísticos de cómo se organiza una elección" (Björnlund, 2004; Muñoz Pogossian y Álvarez Veloso, 2015:59) y con ello poder determinar en qué medida esa elección es buena (o de calidad).

6. Un proyecto para establecer en qué medida las elecciones son libres y justas ha sido el denominado National Elections Across Democracy and Autocracy (NELDA), que cubre 


\section{Flavia Freidenberg}

diversos atributos, como boicots o protestas así como también evalúa las intenciones de los gobernantes de abandonar el poder.

7. Esos informes han recibido críticas, dado que tendían a oscurecer más que aclarar lo que se quería observar (Bjornlund, 2004:95; Norris, 2013:564), empleando "un lenguaje diplomático" (Norris, 2013:564), poco profesional, con respuestas "simplistas, vagas... y políticas" (Bjornlund, 2004:94) y generadas por observadores comprometidos con quienes les invitaron a observar (Bjornlund, 2004:95).

8. La base de datos de calidad de las elecciones provee información a partir de una multitud de fuentes tales como Elections Today, artículos de diversas revistas académicas como Journal of Democracy o Electoral Studies. También incluye la base de datos Lexis Nexis, la base de datos sobre las Instituciones Políticas del Banco Mundial, la Base de Datos Elecciones Africanas, la Election Guide (IFES), Elecciones La vuelta al mundo (Electionworld 2010), Recursos Electorales en Internet, el Archivo Electoral de Adam Carr, la base de datos Parline (Unión Interparlamentaria), entre otros.

9. Esta investigación explora el marco legal y sus deficiencias, la administración pre-electoral, el nivel de violencia, los límites al alcance y a la jurisdicción de los cargos electivos, el uso indebido de fondos públicos, restricciones a la libertad de hacer campaña, a los medios de comunicación, las diferentes formas de intimidación a las candidaturas y/o a los votantes, los problemas en el registro y/o padrón electoral, quejas respecto al modo en que el órgano electoral conduce la información y en el ejercicio del voto, la manipulación del voto, el fraude electoral o la compra de voto, entre otros.

10. Diversas iniciativas trabajan con el concepto de "integridad de las elecciones" como la Comisión Global sobre Elecciones, Democracia y Seguridad; International IDEA, el Proyecto de Elecciones de Integridad de Pippa Norris y su equipo desde Harvard y la Universidad de Sidney (Norris, 2014; Norris et al., 2014) y también la plataforma on line ACE Project [Red de Conocimientos Electorales] o el National Democratic Institute (Merloe, 2015).

11. Este proceso está integrado por diversas fases que se van dando en el marco de un ciclo, como la preelectoral (que incluye la campaña y el modo en que se da la dinámica del proceso), el día "e" (que supone todo los aspectos vinculados al modo en que se hace efectiva la elección) y la post-electoral, que incluye el modo en que los actores políticos y los ciudadanos aceptan (o no) los resultados de las elecciones (Norris, 2013).

12. La base de dichas normas globales está en el artículo 21.3 de la Declaración Universal de los Derechos Humanos de 1948, constituyendo la base jurídica y los principios fundamentales que legitiman el apoyo internacional a las elecciones y la asistencia electoral. Los acuerdos sobre las normas globales que deben regir la conducta de las elecciones se detallan en el artículo 25 del Pacto Internacional de las Naciones Unidas para los Derechos Civiles y Políticos (1966). Ver la Resolución de la Asamblea General de las Naciones Unidas 64 / 155 del 8 de marzo de 2010 sobre el “Fortalecimiento del papel de las Naciones Unidas para mejorar las elecciones periódicas y genuinas y la promoción de la democratización".

13. Nohlen (2015:5) sostiene que es más factible evaluar la integridad de las elecciones por su negativo: "sino se lesionan las normas, si no se manipulan los elementos del 
proceso electoral en contra de lo legalmente o constitucionalmente establecido, [...] si no se contradice, más allá de las normas, a los valores que las sustentan".

14. Se construyó un Índice de Manipulación Electoral, que incluye 15 indicadores, para medir tres dimensiones -la manipulación de las reglas, de los votantes y de la votación-en los países de Europa Central y del Este y algunos países de América Latina y África entre 1995 y 2006. La codificación de los indicadores y la recolección de los datos se realiza a partir de los Informes de las Misiones de Observación Electoral (como las de la Organización para la Seguridad y la Cooperación en Europa, la Unión Europea o la Organización de los Estados Americanos) (Birch, 2015:8).

15. En 1881 el astrónomo y matemático Simon Newcomb descubrió que los dígitos iniciales significativos de los números (i.e. excluyendo el cero) no se distribuían de manera uniforme. En 1938 el físico Frank Benford redescubrió el fenómeno en 20 muestras de diferentes fuentes, que se aportó evidencia rigurosa sobre la presencia recurrente de la distribución logarítmica de los dígitos. Este tipo de análisis se ha aplicado para detectar fraudes fiscales $\mathrm{y}$, en las últimas décadas, para identificar fraudes electorales (Mebane, 2006).

16. Por lo general, este procedimiento estudia el primer o segundo dígito inicial en la cuenta de votos recibidos por cada candidatura, ya sea en la casilla o en las secciones electorales (Mebane, 2006). Cuando los valores extremos no son normales parecen indicar anomalías e irregularidades que se piensan que surgen de prácticas como el fraude electoral, la negligencia, el soborno o, la compra o la manipulación de votos.

17. "[...] Una buena democracia o una democracia de calidad, esa ordenación institucional estable que a través de instituciones y mecanismos que funcionan correctamente, realiza la libertad y la igualdad de los ciudadanos. Entonces, una buena democracia es, antes que todo, un régimen ampliamente legitimado y, por lo tanto, estable, del cual los ciudadanos están plenamente satisfechos (se da una calidad con respecto al resultado) [....] Además, sus ciudadanos y la o las asociaciones y comunidades que forman parte gozan en medida superior a los mínimos de libertad e igualdad (se da una calidad con respecto al contenido) [...] los ciudadanos de una buena democracia deben poder controlar y evaluar si y cómo esos dos valores se realizan a través del pleno respeto de las normas vigentes, el llamado rule of law, su eficiente aplicación, la eficacia en la toma de las decisiones junto a la responsabilidad política por las elecciones tomadas por el personal elegido también en relación a las preguntas expresadas por la sociedad civil (se da una calidad respecto al procedimiento)" (Morlino, 2007:5-6).

18. La confianza política es el activo que los individuos depositan en las instituciones y actores políticos, por eso es clave para conocer el modo en que la ciudadanía se relacionan con ellas. La confianza puede ser de dos tipos: explícita (trust) e implícita (confidence) (Luhmann, 2000). La primera se deposita en una persona o institución en el momento de tomar decisiones que implican un riesgo y la segunda ocurre de manera rutinaria, sin reflexión de por medio, y se deposita igualmente en personas o instituciones. La confianza explícita es la que se da cuando se toma una decisión concreta, por ejemplo, se emite el voto y se apoya a algún candidato. En ese acto, existe una confianza implícita mayor, más allá del acto de votar, pues al participar se está expresando confianza en el régimen político.

19. Sostienen que los observadores realizan pronunciamientos sobre la base de pruebas incompletas (Norris, 2013; Beaulieu y Hyde, 2009; Goodwin-Gill, 2005:85); a partir 


\section{Flavia Freidenberg}

de datos recogidos sólo el día de la votación (Carothers, 1997) y sus recomendaciones pueden ser discutibles cuando las organizaciones llegan a conclusiones opuestas (Daxecker y Schneider, 2014). Muchas veces algunos observadores suelen responder a la convocatoria de sus colegas de los organismos electorales, sujetos a la "política de la conveniencia" (Elklit y Reynolds, 2005a:147), dependiendo de la invitación de un gobierno para acompañar la elección, haciendo que sus declaraciones tengan consideraciones muchas veces más políticas que técnicas. Algunos reportes están condicionados por las relaciones de los observadores con sus pares, sujetos a ser invitados por los gobiernos (lo que genera que haya países que no puedan estudiarse porque no reciben observadores) o incluso sobre la base de no poder denunciar aspectos técnicos concretos.

20. Según la base de datos de la OEA, se han realizado 122 misiones (mientras que en 1993-2002, fueron 66; entre 1983-1992, unas 23; entre 1973-1982, 7 y entre 1962 y 1972, 12). Ver Muñoz Pogossian y Álvarez Veloso (2015:64). Por su parte, la Unión Europea ha enviado veintiún Misiones de Observación Electoral entre 2000 y 2014 a diez países de América Latina. Datos relevados el 27 de febrero de 2017 de la Página web del Departamento de Observación y Capacitación Electoral. Disponible aquí: http:/ / www.oas.org/eomdatabase/default.aspx?lang=es

21. Esto es así dado que resulta complejo descontextualizar las elecciones de la dinámica del régimen político (Diamond, 2004; Levitsky y Way, 2004) o del propio funcionamiento del sistema político (Nohlen, 2015). Como sostiene en reiteradas oportunidades Nohlen (2015), "el contexto hace la diferencia".

22. La encuesta de Latinobarómetro 2015 se aplicó 20, 250 entrevistas cara a cara en 18 países entre el 15 de enero y el 15 de febrero. En México se realizó entre el 8 y el 24 de noviembre de 2014 y en Ecuador entre el 10 de agosto y el 2 de septiembre de 2015. Las muestras son representativas al 100 por ciento de la población nacional de cada país considerando 1000 y 1200 casos, con un margen de error de alrededor del 3\% por país (Latinobarómetro, 2015).

23. Esto hace menos probable que su partido o su candidato/a presidente gane la elección cuando se enfrenta a un incumbent, ya que esta condición aumenta un 62 por ciento la probabilidad de ganar una elección (Penfold et al., 2014).

24. El Informe en Perú recomendó: “Con el fin de mejorar la participación de las mujeres en la contienda, ... incluir la obligatoriedad de la alternancia por sexo en la conformación de las listas... también sugiere que el Congreso discuta el proyecto de "Ley contra el acoso político hacia las mujeres" que permitirá hacer frente a uno de los obstáculos que enfrentan a la hora de participar en el espacio público" (iMOE/OEA Perú 2014: 5).

25. Sostiene el Informe: “[...] de los trece partidos que participaron en la contienda, cinco concentraron el $88 \%$ de la cobertura informativa, y sólo cuatro concentraron el $80 \%$ de la pauta publicitaria. La inequidad reflejada en estas cifras se profundizó en el tratamiento de las mujeres candidatas, quienes estuvieron virtualmente invisibilizadas en los medios de comunicación. [...] las mujeres candidatas a la Asamblea recibieron el $22 \%$ de la cobertura [...] contrastado con el $78 \%$ dedicado a las candidaturas masculinas" (iMOE/OEA Costa Rica 2014:2). 
26. El Informe de Costa Rica sostiene que " 5 de los 13 partidos políticos a la Presidencia utilizaron sólo el $7.25 \%$ del total de fondos que tenían disponibles para este fin" (iMOE/OEA Costa Rica 2014:3).

27. El Informe recomienda establecer en la Ley de Partidos, los “topes ... en el gasto publicitario. Asimismo, explicitar los mecanismos de rendición de cuentas, y dotar al Tribunal Supremo Electoral de competencias para el control del financiamiento de partidos y candidatos" (iMOE/OEA El Salvador 2015:5).

28. La Misión de Observación de la OEA sugirió “[...] la revisión a la legislación, de manera de que no se generen incentivos a la excesiva litigiosidad que se ha producido en este proceso electoral. [se] sugiere se revise el esquema jurisdiccional implementado a partir de la creación de la Sala Especializada del TEPFJ, racionalizando las funciones y evitando duplicidades y contradicciones con otras instancias. [y] de la importancia de delimitar claramente roles, funciones y competencias diferenciadas de forma que se garantice una relación más fluida entre el INE y los organismos públicos locales electorales (OPLEs)" (iMVE México 2015:3).

29. En el caso de Honduras, se recomendó claramente que se realizara la reforma "de la composición partidaria del órgano electoral en todos sus niveles de jerarquía, a saber sus magistrados, su cuerpo de funcionarios y los miembros de las mesas de votación, a fin de transitar hacia un servicio civil profesional en la institución" [iMOE Honduras 2013:38]. 


\section{REFERENCIAS BIBLIOGRÁFICAS}

AZPURU, Dinorah. (2015), La Legitimidad de la Democracia a la Luz de su Desempeño: Una Evaluación de las Preferencias Ciudadanas (1994-2014). Seminario Internacional: Dilemas de la Democracia en América Latina: A Cuatro Décadas de la Transición, IIJ-UNAM y FLACSO, Ciudad de México, 30 de septiembre-2 de octubre.

BEAULIEU, Emily; HYDE, Susan D. (2009), "In the Shadow of Democracy Promotion: Strategic Manipulation, International Observers, and Election Boycotts". Comparative Political Studies, vol. 42, pp. 392-415.

BIRCH, Sarah. (2008), “Conceptualising Electoral Malpractice. Project on Electoral Malpractice in New and Semi-Democracies". Working Paper nํ 1. http://www.essex. ac.uk/government/electoralmalpractice. Consultado el 15 de marzo de 2017.

. (2011), Electoral Malpractice. Oxford, Oxford University Press.

. (2015), "Patterns of Electoral Malpractice. Project on Electoral Malpractice in New and Semi-Democracies". Working Paper no 4. http://www.essex.ac.uk/government/electoralmalpractice/. Consultado el 30 de mayo de 2016.

BJÖRNLUND, Eric C. (2004), Beyond Free and Fair: Monitoring Elections and Building Democracy. Washington D.C., Woodrow Wilson Center Press y Johns Hopkins University Press.

CAROTHERS, Thomas. (1997), “The Rise of Election Monitoring. The Observers Observed". Journal of Democracy, vol. 8, no 3, pp. 17-31.

CARRERAS, Miguel; IREPOGLU, Yasemin. (2013b), "Perceptions of Electoral Integrity, Efficacy and Electoral Participation in Latin America". Electoral Studies, vol. 32, oㅡ 4, pp. 600-619.

CORDENILLO, Raul; REYNOLDS, Andrew (eds.). (2012), The Integrity of Elections. The Role of Regional Organizations. Estocolmo, International Institute for Democracy and Electoral Assistance.

DAXECKER, Ursula; SCHNEIDER, Gerald. (2014), “Election Monitoring: The Implications of Multiple of Monitors for Electoral Integrity", en P. Norris; R. W. Frank; F. Martínez i Coma (eds.), Advancing Electoral Integrity. Oxford, Oxford University Press, pp. 73-93.

DAHL, Robert. (1971), La Poliarquía. Madrid, Tecnos.

DIAMOND, Larry. (2004), “Elecciones sin Democracia. A Propósito de los Regímenes Híbridos". Estudios Políticos, vol. 24, pp. 117-134.

DONNO, Daniela. (2010), “Who Is Punished? Regional Intergovernmental Organizations and the Enforcement of Democratic Norms". International Organization, vol. 64, pp. 593-625.

ELKLIT, Jørgen; REYNOLDS, Andrew. (2005a), “A Framework for the Systematic Study of Election Quality". Democratization, vol. 12, no 2, pp. 147-162.

. (2005b), “Judging Elections and Election Management Quality by Process”. Representation, vol. 41, no 3, pp. 189-207. 
FREIDENBERG, Flavia. (2013), “La Calidad de las Elecciones en América Latina”. Mundo Electoral, Año 6 [18]. Disponible en http:/ / www.mundoelectoral.com/html/index.php?id=1138. Consultado el 30 de diciembre de 2015.

; CISNEROS, Gerardo Isaac. (2016), “Confianza”. Revista Voz y Voto, vol. 279, pp. 40-44.

FREIDENBERG, Flavia; DOŠEK, Tomás. (2016), “Las Reformas Electorales en América Latina [1978-2015]", en K. C. Zamora et al. (eds.), Reformas Políticas en América Latina: Tendencias y Casos. Washington, D.C., Organización de los Estados Americanos.

FREIDENBERG, Flavia; SUÁREZ-CAO, Julieta (eds.). (2014), Territorio y Poder: Nuevos Actores y Competencia Política en los Sistemas de Partidos Multinivel en América Latina. Salamanca, Ediciones Universidad de Salamanca.

GOODWIN-GIL, Guy S. (2005), Elecciones Libres y Justas. Ginebra, Unión Interparlamentaria.

HARTLYN, Jonathan; McCOY, Jennifer. (2001), Elections with "Adjectives" in Contemporary Latin America: A Comparative Analysis. XXIII Congreso Internacional de LASA, Washington, D.C.

(2006), "Observer Paradoxes: How to Assess Electoral Manipulation", en A. Schedler (ed.), Electoral Authoritarianism: The Dynamics of Unfree Competition. Miami, Lynne Rienner Publisher, pp. 41-56.

HARTLYN, Jonathan; MCCOY, Jenniffer; MUSTILLO, Tom. (2008), “Electoral Governance Matters: Explaining the Quality of Elections in Contemporary Latin America". Comparative Political Studies, vol. 41, no 1, pp. 73-98.

HYDE, Susan D. (2011), The Pseudo-Democrats' Dilemma. Ithaca, Cornell University Press.

JIMÉNEZ, Raúl; HIDALGO, Manuel. (2014), “Forensic Analysis of Venezuelan Elections during the Chávez Presidency". Plos One, vol. 9, no 6, pp. 1-18.

KELLEY, Judith. (2010), "Election Observers and their Biases". Journal of Democracy, vol. 21, no 3, pp. 158-172.

. (2011), "Do International Election Monitors Increase or Decrease Opposition Boycotts?". Comparative Political Studies, vol. 44, no 11, pp. 1527-1556.

; KOLEV, Kiril. (2010), “Elections Quality and International Observation: 1975-2004. Two New Datasets". Disponible en SSRN: http://ssrn.com/abstract= 1694654. Consultado el 20 de diciembre de 2015.

LEEMAN, Lucas; BOSCHLER, Daniel. (2014), “A Systematic Approach to Study Electoral Fraude". Electoral Studies, vol. 35, pp. 33-47.

LEHOUCQ, Fabrice. (2002), “Can Parties Police Themselves? Electoral Governance and Democratization". International Political Science Review, vol. 23, no 1, pp. 29-46.

LEVITSKY, Steven; WAY, Lucas. (2004), “Elecciones sin Democracia: El Surgimiento de los Regímenes Autoritarios Competitivos". Estudios Políticos, vol. 24, pp. 159-176.

LINZ, Juan J. (1987), La Quiebra de las Democracias. Madrid, Alianza Editorial.

LUHMANN, Niklas. (2000), “Familiarity, Confidence, Trust: Problems and Alternatives", en D. Gambetta (ed.), Trust: Making and Breaking Cooperative Relations. Oxford, University of Oxford, pp. 94-107. 


\section{Flavia Freidenberg}

MARTÍNEZ I COMA, Ferrán; NORRIS, Pippa; FRANK, Richard W. (2015), “Integridad Electoral en América Latina 2012-2014". América Latina Hoy, Revista de Ciencias Sociales, vol. 70, pp. 37-54.

MEBANE, Walter Jr. (2006), "Election Forensics: Vote Counts and Benford's Law", Working Paper, Department of Political Science, University of Michigan.

MELIÁN, Xavier; TUCCINARDI, Doménico. (2015), “La Observación Electoral Europea en América Latina. Un Análisis de 15 Años de Recomendaciones (2000-2014)". América Latina Hoy, Revista de Ciencias Sociales, vol. 70, pp. 77-90.

MÉNDEZ DE HOYOS, Irma. (2013), A Preliminary Framework to Analyse the Quality of Elections in Latin America: Mal Practices in Presidential Elections 2006-2012". Ponencia presentada en el VII Congreso del Consejo Europeo de Investigaciones Sociales sobre América Latina, Oporto, 12-15 de junio.

MERLOE, Patrick. (2015), “Electoral Monitoring vs. Disinformation”. Journal of Democracy, vol. 26, no 3, pp. 79-93.

MORLINO, Leonardo. (2007), “Explicar la Calidad Democrática: ¿Qué tan Relevantes son las Tradiciones Autoritarias?". Revista de Ciencia Política, vol. 27, no 2, pp. 3-22.

MOZAFFAR, Shaheen; SCHEDLER, Andreas. (2002), “The Comparative Study of Electoral Governance: Introduction". International Political Science Review, vol. 23, no 1, pp. 5-27.

MUÑOZ-POGOSSIAN, Betilde; ÁLVAREZ VELOSO, David. (2015), “La Responsabilidad De Observar: Repensando la Observación Electoral de la OEA". América Latina Hoy, Revista de Ciencias Sociales, vol. 70 (agosto), pp. 55-76.

NOHLEN, Dieter. (2015), “Arquitectura Institucional, Contexto Sociocultural e Integridad Electoral". Documento presentado en la Segunda Asamblea General de la Association of World Elections Bodies (AWEB), Punta Cana, República Dominicana, 20 de agosto.

NORRIS, Pippa. (2013), “The New Research Agenda Studying Electoral Integrity”. Electoral Studies, vol. 32, pp. 563-575.

. (2014), Why Electoral Integrity Matters. New York, Cambridge University Press.

; FRANK, Richard; MARTÍNEZ I COMA, Ferran. (2014), "Measuring Electoral Integrity around the World: A New Dataset". Political Science \& Politics, vol. 47, oㅡ 4, pp. 789-798.

PASTOR, Robert A. (1998), “Mediating Elections”. Journal of Democracy, vol. 9, no 1, pp. 154-163.

PENFOLD, Michael; CORRALES, Javier; HERNÁNDEZ JIMÉNEZ, Gonzalo. (2014), "Los Invencibles: La Reelección Presidencial y los Cambios Constitucionales en América Latina". Revista de Ciencia Política, vol. 34, no 3, pp. 537-539.

SCHATTSCHNEIDER, Elmer E. (1942), Party Government. New York, Holt, Rinehart and Winston. [Régimen de Partidos. Madrid, Editorial Tecnos, 1964].

SCHEDLER, Andreas. (2002), "Elections without Democracy: The Menu of Manipulation". Journal of Democracy, vol. 13, no 2, pp. 36-50. 
(2013), The Politics of Uncertainty: Sustaining and Subverting Electoral Authoritarianism. Oxford, Oxford University Press.

ZOVATTO, Daniel. (2006), “Elecciones y Democracia en América Latina. Balance Electoral Latinoamericano Noviembre 2005-Diciembre 2006". Ponencia presentada en el Seminario de Balance Electoral de América Latina, organizado en el Centro de Estudios Constitucionales, Madrid, 13 de diciembre.

\section{Informes de las Misiones de Observación Electoral 2013-2016}

Bolivia 2014. Informe Verbal de la Misión de Observación Electoral en las Elecciones Generales del 12 de octubre de 2014 del Estado Plurinacional de Bolivia.

Bolivia 2015. Informe ante el Consejo Permanente de la Misión de Observación Electoral en las Elecciones Subnacionales del 29 de marzo de 2015 en el Estado Plurinacional de Bolivia.

Colombia 2014. Informe Verbal de la Misión de Veeduría Electoral en las Elecciones legislativas y presidenciales (primera y segunda vuelta) del 2 de marzo, 25 de mayo y 15 de junio de 2014 de la República de Colombia.

Colombia 2015. Informe ante el Consejo Permanente de la Misión de Veeduría Electoral (MVE) en las Elecciones de Autoridades Locales del 25 de octubre de 2015.

Costa Rica 2014. Informe Verbal de la Misión de Observación Electoral en las Elecciones Generales del 2 de febrero y del 6 de abril de 2014 de la República de Costa Rica.

Costa Rica 2016. Informe ante el Consejo Permanente de la Misión de Observación Electoral en las Elecciones Municipales del 7 de febrero de 2016 en la República de Costa Rica.

Ecuador 2013. Informe Verbal de la Misión de Observación Electoral en las Elecciones Generales del 17 de febrero y 7 de abril de 2013 de la República de Ecuador.

Ecuador 2014. Informe Verbal de la Misión de Observación Electoral en las Elecciones Municipales del 23 de febrero de 2014 de la República de Ecuador.

El Salvador 2015. Informe ante el Consejo Permanente de la Misión de Observación Electoral a las Elecciones Asamblea Legislativa, Parlamento Centroamericano y Concejos Municipales del 1 de marzo de 2015 de la República de El Salvador.

Honduras 2013. Informe Final de la Misión de Observación Electoral de la Organización de los Estados Americanos, Elecciones Generales del 24 de noviembre de 2013 de la República de Honduras.

México 2015. Informe ante el Consejo Permanente de la Misión de Visitantes Extranjeros en las Elecciones Federales del 7 de junio de 2015 de los Estados Unidos Mexicanos.

Panamá 2014. Informe Verbal de la Misión de Observación Electoral en las Elecciones Generales del 4 de mayo de 2014 de la República de Panamá.

Paraguay 2013. Informe de la Misión de Observación Electoral de la Organización de los Estados Americanos, Elecciones Generales del 21 de abril de 2013 de la República de Paraguay.

Paraguay 2015. Informe ante el Consejo Permanente de la Misión de Observación Electoral en las Elecciones Municipales del 15 de noviembre de 2015 en Paraguay. 


\section{Flavia Freidenberg}

Perú 2014. Informe Verbal de la Misión de Observación Electoral en las Elecciones regionales y Municipales del 5 de octubre de 2014 de la República de Perú.

Perú 2016. Informe de la Misión de Observación de la OEA para las elecciones en Perú recomienda profunda reforma electoral del 11 de abril de 2016.

República Dominicana 2016. Informe ante el Consejo Permanente. Misión de Observación Electoral en las Elecciones Ordinarias Generales Presidenciales, Congresuales, Municipales y de Representantes de la comunidad Dominicana en el Exterior, del 15 de mayo de 2016. 


\section{RESUMO}

O que Seria uma Boa Eleição? O Uso dos Informes das Missões de Observação Eleitoral na Avaliação dos Processos Eleitorais

Latino-americanos (2013-2016)

Ainda que as eleições sejam o coração da democracia representativa, não existe consenso acerca do que é uma boa eleição e tampouco há garantias que as práticas políticas desenvolvidas atendam a critérios de qualidade, legitimidade e credibilidade, em conformidade com os parâmetros internacionalmente estabelecidos. Ademais, em muitos países considerados democráticos, as eleições envolvem irregularidades, práticas incorretas e problemas de governança eleitoral. Este artigo define uma "boa eleição" como aquela que satisfaz três requisitos imperativos: a) respeito dos padrões internacionais de integridade eleitoral (procedimento); b) garantia do exercício de uma série de direitos políticos (conteúdo); e c) satisfação das expectativas dos cidadãos com respeito à forma de organização das eleições e ao alcance dos seus objetivos (resultado). Após avaliação dos Relatórios das Missões de Observação Eleitoral da Organização dos Estados Americanos referentes a 18 eleições na América Latina (2013-2016), evidencia-se que, embora os níveis de qualidade das eleições tenham sido elevados em duas dimensões-chave (organização técnica e respeito pelos direitos políticos), ainda persistem problemas em termos de resultados, assim como em relação ao conteúdo.

Palavras-chave: eleições; missões internacionais de observação; integridade eleitoral; Organização dos Estados Americanos; América Latina

\section{ABSTRACT \\ What Constitutes a Good Election? Employing Reports on Electoral Observation Missions to Evaluate Latin American Electoral Processes (2013-2016)}

Although elections lie at the heart of representative democracy, a lack of consensus remains on what constitutes a good election, as do guarantees that the political practices developed meet quality, legitimacy, and credibility criteria set by internationally-established parameters. Elections in many countries considered to be democratic are also rife with irregularities, cases of malpractice, and problems with electoral governance. The following article suggests defining a "good election" as one which necessarily satisfies three conditions: a) respect for the international standards of electoral integrity (procedure); b) a guaranteeing of the exercise of a series of political rights (content), and c) a satisfying of citizens' expectations on the way in which elections are organized and their aims achieved (result). By means of an evaluation of Reports on Electoral Observation Missions (2013-2016) carried out by the Organization of American States, we demonstrate that although election quality levels increased in the period for two key facets (technical organization and that regarding political rights), problems remain in terms of the results, as well as in terms of content.

Key words: elections; international observation missions; electoral integrity; Organization of American States; Latin America 
RÉSUMÉ

Qu'est-ce qu'une Bonne Élection? L'Usage des Rapports des Missions

d'Observation Électorale pour Évaluer les Processus Électoraux

Latino-américains (2013-2016)

Même si les élections constituent le cœur de la démocratie représentative, il n'existe néanmoins aucun consensus quant à ce qui constituerait une bonne élection et rien ne garantit que les pratiques politiques en œuvre répondent aux critères de qualité, légitimité et crédibilité promus au niveau international. En effet, dans de nombreux pays considérés comme démocratiques, les élections sont souvent l'occasion d'irrégularités, de mauvaises pratiques et autres problèmes de gouvernance électorale. Cette article propose de définir une «bonne élection » comme un scrutin à même de satisfaire trois conditions préalables : a) le respect des normes internationales d'intégrité électorale (procédure) ; b) la garantie de l'exercice d'une série de droits politiques (contenu), et c) la satisfaction des attentes citoyennes quant à la façon dont sont organisées les élections et atteints leurs objectifs (résultat). À partir de l'évaluation des rapports des Missions d'observation électorale de l'Organisation des États américains relatifs à dix-huit scrutins tenus en Amérique latine (2013-2016), on a mis en évidence qu'en dépit de l'augmentation des niveaux de qualité des élections quant à deux dimensions clés (organisation technique et respect des droits politiques), il existe encore de nombreux problèmes quant aux résultats ainsi qu'au contenu.

Mots-clés: élections; Missions internationales d'observation; intégrité électorale; Organisation des États américains; Amérique latine

\section{RESUMEN}

¿Qué es una Buena Elección? El Uso de los Informes de las Misiones de Observación Electoral para Evaluar los Procesos Electorales

Latinoamericanos (2013-2016)

Aún cuando las elecciones son el corazón de la democracia representativa, todavía no existe consenso respecto a qué es una buena elección ni tampoco se ha garantizado que las prácticas políticas que se desarrollan cumplan con criterios de calidad, legitimidad y credibilidad siguiendo los parámetros establecidos internacionalmente. En muchos países considerados democráticos las elecciones conviven con irregularidades, malas prácticas y problemas de gobernanza electoral. Este artículo propone definir una "buena elección" como aquella que satisface tres condiciones necesarias: a) respeta los estándares internacionales de integridad electoral (procedimiento); b) garantiza el ejercicio de una serie de derechos políticos (contenido) y c) satisface las expectativas de la ciudadanía respecto al modo en que se organizan las elecciones y se consiguen sus objetivos (resultado). Tras evaluar los Informes de las Misiones de Observación Electoral de la Organización de los Estados Americanos de 18 elecciones en América Latina (2013-2016), se evidencia que aunque se han incrementado los niveles de calidad de las elecciones en dos dimensiones claves (organización técnica y respeto a los derechos políticos), aún existen problemas en cuanto a los resultados así como también en relación al contenido.

Palabras clave: elecciones; misiones de observación internacional; integridad electoral; Organización de los Estados Americanos; América Latina 